\title{
AUTOMATED MODAL IDENTIFICATION AND TRACKING: APPLICATION TO AN IRON ARCH BRIDGE
}

\begin{tabular}{|r|l|}
\hline Journal: & Structural Control and Health Monitoring \\
\hline Manuscript ID & STC-15-0182.R1 \\
\hline Wiley - Manuscript type: & Research Paper \\
\hline Date Submitted by the Author: & 04-Dec-2015 \\
\hline Complete List of Authors: & $\begin{array}{l}\text { Cabboi, Alessandro; University of Cambridge, Engineering Department } \\
\text { Magalhães, Filipe; University of Porto, Civil Engineering; } \\
\text { Gentile, Carmelo; Politecnico di Milano, Department of Architecture, Built } \\
\text { environment and Construction engineering (ABC) } \\
\text { Cunha, Alvaro; Faculty of Engineering of University of Porto (FEUP), Civil } \\
\text { Engineering }\end{array}$ \\
\hline Keywords: & $\begin{array}{l}\text { Automatic modal identification, Vibration monitoring, Operational modal } \\
\text { analysis, Structural dynamics, Iron arch bridge }\end{array}$ \\
\hline
\end{tabular}

\section{SCHOLARONE ${ }^{m}$}

Manuscripts 
AUTOMATED MODAL IDENTIFICATION AND TRACKING: APPLICATION TO AN IRON ARCH BRIDGE

Keywords: Automatic modal identification, Vibration monitoring, Operational modal analysis, Structural dynamics, Iron arch bridge

\begin{abstract}
Challenges concerning the automation of modal identification and tracking procedures in permanent monitoring systems for Structural Health Monitoring purposes are discussed. In this context, an automated procedure based on parametric identification methods that involve the interpretation of stabilization diagrams is proposed. The methodology comprehends two key points: (a) automatic analysis of stabilization diagrams, performed through a first check of reasonable damping ratio, a subsequent modal complexity check and a final clustering of structural modes; (b) automated tracking of the evolution in time of the identified modal properties. The proposed modal clustering and tracking steps exploit the introduction of self-adaptable dynamic thresholds, that do not require any a priori manual tuning for the different recorded data set. Finally, the proposed approach was successfully validated using real data collected on a historic iron arch bridge.
\end{abstract}

\title{
1. INTRODUCTION
}

In the last decade, the application of vibration-based Structural Health Monitoring (SHM) has seen an increasing development in the area of Civil Engineering structures [1-3]. Ageing of existing infrastructures (often accompanied by poor maintenance and harsh environmental conditions), increasing complexity of new constructions and the possible occurrence of exceptional loads provide strong motivations to the research effort around this topic. Furthermore, the minimum impact of the vibration monitoring makes this technology useful also in the context of preservation of Cultural Heritage structures [4-6].

Within this framework, since the first goal of dynamic monitoring is to continuously provide an image about the dynamic characteristics of the structure at different times, an important role is played by the development of automatic modal identification algorithm apt 
to efficiently handle the continuously recorded vibration responses. Several procedures described in the literature are based on parametric identification methods developed in the time domain [7-9] such as the Stochastic Subspace Identification (SSI) techniques [10,11] that offer a high accuracy in the identification of closely-spaced modes and are especially suited to be automated due to their algebraic nature - or in the frequency domain [12] like the poly-Least Squares Complex Frequency Domain method (Poly-MAX) [13] recognized for its stable identification of the system poles leading to clearer stabilization diagrams. Concurrently, other procedures are based on non-parametric identification techniques often developed in the frequency domain [14,15] such as the Frequency Domain Decomposition method (FDD) [16] which is well-known for the simplicity and its more strict physical meaning.

The present work is focused on the development of an automated procedure apt to manage the results obtained from any parametric identification method that require the interpretation of stabilization diagrams. The performance of the proposed procedure is exemplified using the Covariance-based Stochastic Subspace Identification (SSI-Cov) technique. Generally speaking, a fully automated modal identification methodology based on parametric methods can be briefly summarized in four steps:

(a) digital signal pre-processing of the recorded data;

(b)modal parameter identification using models with varying dimensions;

(c)automatic analysis of the stabilization diagram. This step can further be divided in elimination of noise modes (optional) and clustering of physical modes to obtain the most representative values of the estimated parameters of each clustered mode: natural frequency, damping and mode shape.

(d)modal tracking, that tracks and records the time evolution of the previous identified modes by iteratively repeating the first three steps for subsequent recorded vibration responses.

The proposed procedure introduces new aspects and further observations to step (c) and (d), which are widely recognized to be the most challenging tasks. More specifically, the main goal of this work is to automate the decision process of an experienced-human operator regarding the selection (c) and tracking (d) of the most meaningful identified modes for SHM purposes. In this context, the main contributions are the development of:

1) a clustering algorithm that automatically interprets stabilization diagrams. In this stage, the target is to minimize any manual tuning of user-defined parameter, in order to make the algorithm suitable for any parametric identification method that relies on stabilization diagrams. Furthermore, if compared to existing clustering methods that entirely $[8,17]$ or partially $[7,12]$ fits the above statement, the proposed algorithm is computationally less demanding;

2) a complete automated modal tracking algorithm. Usually, tracking and pairing the most significant modes from different data-sets requires a baseline list of pre-selected vibration modes of the investigated structure and a set of indices and static thresholds in order to define which modes share the same modal properties [7,12]. Since natural frequencies of the modes may change due to variation in the operating condition (e.g. temperature variation [3]), it is often necessary to manually update such thresholds. On the contrary, the proposed procedure is based on self-adaptable thresholds avoiding any user interaction in such process. 
In order to demonstrate the performance of the proposed methodology, the current approach is exemplified using data continuously collected during one month (from 27 February to 1 April 2012) by the dynamic monitoring system installed on the historic San Michele bridge (1889) [15], one of the most important monuments of XIX century iron heritage [18-21]. For validation purposes, the estimates of the natural frequencies of vertical and transversal modes - provided by the proposed procedure applied with the SSI-Cov method - are firstly compared to colour maps illustrating the time evolution of the first singular values of the estimated spectral matrix for each data set. Since the estimation of the vertical modes of the current case study has been revealed to be quite challenging, a further validation is presented comparing the obtained results to those independently identified by a manual interpretation of 723 stabilization diagrams produced by the Data-driven SSI technique. It is worth mentioning that the latter validation approach is quite unique in the literature and permits an effective evaluation of the automated algorithm, especially regarding steps (c) and (d).

It is further noticed that the quality of the clustering results and consequently of the modal estimates often depends on the proper selection of the input parameters of the modal identification algorithm. This aspect is discussed in section 3, but an exhaustive analysis falls outside the scope of the current work.

\section{THE SAN MICHELE BRIDGE AND ITS MONITORING SYSTEM}

The San Michele bridge is an iron arch bridge that crosses the Adda river, linking the small towns of Paderno and Calusco d'Adda, about $50 \mathrm{~km}$ far from Milan. The bridge, built in 1889 by the Società Nazionale delle Officine di Savigliano $[18,19]$ in order to complete one of the first Italian railway lines, is still used as a combined road and railway bridge.

As shown in Fig. 1, the bridge is characterized by the main parabolic iron arch, the upper truss box metal girder and its supporting piers. The arch spans $150 \mathrm{~m}$ and rises $37.5 \mathrm{~m}$. It is composed by two ribs that are canted inward with a distance of about $16 \mathrm{~m}$ at the basement and $5 \mathrm{~m}$ at the crown. The upper girder is $266 \mathrm{~m}$ long and it is supported by nine equally spaced bearings. Two bearings are placed over the abutments, three are placed on the top of three piers built on masonry basements and the remaining four bearings are placed on the top of four piers supported by the main arch. The main girder consisting of two vertical trusses (6.25 $\mathrm{m}$ high and $5.0 \mathrm{~m}$ apart) support two decks: the upper one for alternate road traffic and pedestrian traffic, and the lower for a single rail line. The weight and speed of vehicles are limited: $180 \mathrm{kN} /$ axis and $15 \mathrm{~km} / \mathrm{h}$ for the trains, $35 \mathrm{kN}$ and $20 \mathrm{~km} / \mathrm{h}$ for the road vehicles.

The state of preservation of the bridge is rather poor due to the difficulty of performing a regular maintenance, and a wide number of structural members are significantly damaged by corrosion. Moreover, the bridge has not been saved from the progressive traffic increase, generally experienced by the infrastructures during the last decades. Hence, in order to better understand the dynamic response of the bridge in operational conditions, several ambient vibration tests (AVTs) were performed on the roadway deck between June and October 2009 [20] and on the railway deck, in March 2010 [15] by the Laboratory of Vibrations and Dynamic Monitoring of Structures (VIBLAB) of Politecnico di Milano.

The results of those tests, reported in $[15,20]$, motivated the installation of a permanent 
dynamic monitoring system on the bridge with SHM purposes. In agreement with the Italian Railway Authority (RFI), the main institutional owner of the bridge, it was decided to install the monitoring system on the railway deck, because the installation and subsequent maintenance operation was more practical with the support of RFI technical staff.

The continuous dynamic monitoring system was finally installed on the bridge in Autumn 2011 and is fully active since 28 November 2011 [15]. The system is completely wired and consists of 21 MEMS accelerometers (sensitivity $1200 \mathrm{mV} / \mathrm{g}$, acceleration range $\pm 3 \mathrm{~g}$, noise density $<0.5 \mu \mathrm{grms} / \sqrt{\mathrm{Hz}}$ ), seven data acquisition (DAQ) units, two thermocouples, two Ethernet switch devices and one industrial PC. Three accelerometers, two to measure the vertical accelerations on the downstream and upstream sides and one to measure the lateral acceleration, were mounted in 7 different cross-sections, corresponding to the bearings of the truss-box girder between the abutments (Fig. 2). The two temperature sensors are placed on the second and the fifth cross sections only, one per side in order to measure the air temperature nearby the structure. The two switch devices collect the Ethernet cables from each group of channels and the digitized data are transmitted to an industrial PC on site. A new binary file, containing 21 acceleration time series (sampled at $200 \mathrm{~Hz}$ ) and the temperature data, is created every hour, stored on the local PC and transmitted to Politecnico di Milano.

Before applying any modal identification algorithm, the data files are first pre-processed by the software described in [21]. Since the responses induced by the railway traffic do not comply with some basic assumptions of Operational Modal Analysis (OMA), such as white noise and stationarity, pre-processing is especially aimed at recognizing and extracting the time series associated to the railway traffic, so that one "train-free" dataset per hour, having a duration of $2400 \mathrm{~s}$, is created [21]. Further pre-processing steps include low-pass filtering and decimation of each dataset. Of course, an appropriate software is installed on the local PC as well for the management of the continuous acquisition, the data storage and transmission, and to check the fitness-for-purpose of the monitoring system.

\section{COVARIANCE-DRIVEN STOCHASTIC SUBSPACE IDENTIFICATION: BACKGROUND AND PRACTICAL APPLICATION}

The reference-based version of the SSI-Cov method, used in the current application, is fully described in [11] and well-known to the modal analysis community since more than one decade. Briefly, the SSI-Cov method is based on the classical state-space form of the discretetime equation of motion of a linear, time invariant N-DOF system under white noise excitation:

$$
\begin{aligned}
& \boldsymbol{x}_{k+1}=\boldsymbol{A} \boldsymbol{x}_{k}+\boldsymbol{w}_{k} \\
& \boldsymbol{y}_{k}=\boldsymbol{C} \boldsymbol{x}_{k}+\boldsymbol{v}_{k}
\end{aligned}
$$

where:

- the sampling instant is denoted by $k$;

- $\boldsymbol{A}$ is the $n \times n$ state matrix $(n=2 N)$;

- $\quad C$ is the $l \times n$ output matrix, selecting the $l$ measured signals from the corresponding internal states, collected in the discrete-time state vector $\boldsymbol{x}_{k}$; 
- $\boldsymbol{y}_{k}$ is the measurements vector;

- $\quad \boldsymbol{w}_{k}$ and $\boldsymbol{v}_{k}$ represent the effect of unknown inputs, modelling inaccuracies and measurement noise. These last vectors are assumed to be zero-mean realizations of stationary stochastic processes and independent from the actual state.

Taking into account these assumptions, it has been shown in $[10,11]$ that the modal parameters can be extracted by relying only on the output response which covariance function can be viewed as the free dynamic response of the structure.

In practical terms, the SSI-Cov identification process requires the time-lag $i$ to construct the output covariance functions and the definition of a model order $(n)$ in order to estimate the state space matrix $\boldsymbol{A}$. A reasonable estimate of the model order could be done by taking advantage of the SVD of the Toeplitz matrix (which gathers the different covariance functions of the dynamic response), and looking at a "gap" between two successive singular values. However, when dealing with ambient vibration data, the order determination via SVD is not proven to be an effective method. The most common strategy used to tackle this problem involves the construction of the so-called stabilization diagram (see e.g. Fig. 7), i.e. a plot of all the identified system poles for a predefined range of the model order [11]. System poles which appear in most of these models with consistent modal properties (frequency, damping or mode shape) are considered physical; conversely the poles that appear only in some models in a scattered way are considered spurious. The physical modes should stabilize for increasing model orders and are graphically identifiable along vertical alignments in the stabilization diagram. Generally, the same strategy is followed for other parametric identification methods that require a model order selection, like the p-LSCF method, since it is not possible to anticipate which model order will provide the best results.

The order of the models $(n)$ to be fitted to the experimental data depends on the number of modes expected in the frequency band under analysis, which in the case of the parametric methods in the time domain can only be between zero and the Nyquist frequency. Therefore, in the present application to limit the maximum model order and to focus the analysis on the frequency band of interest the original data sampled at $200 \mathrm{~Hz}$ were low pass filtered and decimated to a new sampling frequency of $40 \mathrm{~Hz}$ for the vertical accelerations and $20 \mathrm{~Hz}$ for the lateral accelerations.

The input parameters for the SSI-Cov algorithm adopted in the current application are: a)

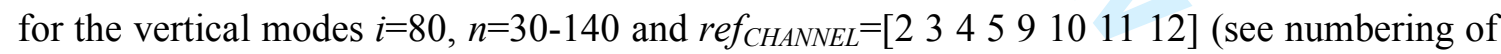
the channels in Figure 2); b) for the transversal modes $i=100, n=50-140$ and $\operatorname{ref}_{\text {CHANNEL }}=[16$ 1718 20]. All parameters needed some previous manual tuning in order to be properly defined. For example, the choice of the time-lag $i$ was driven in both cases by the detection of a stable damping identification for some modes, picking up the minimum value of $i$ that guarantees such condition as illustrated in Figure 3. On the other hand the range of model orders was selected in order to identify even some weakly excited modes, keeping at the same time $n_{\max }$ as low as possible. In fact, higher values of $i$ and $n_{\max }$ can introduce further nonphysical poles, increasing the computational time and the difficulty to distinguish between physical and spurious poles. This can be explained by the fact that high values of $i$ may introduce noise in the output covariance function (2), due to its low amplitudes at higher timelags which contain poor structural information. Therefore, the system model tends to fit the noise content leading to the appearance of more spurious poles in the stabilization diagram. 
On the other hand, $n_{\min }$ can be easily selected. The system poles extracted by the eigenvalue decomposition of the system matrix $\boldsymbol{A}$ appear in conjugate pairs and only the poles with a positive imaginary component are used. This means that $n_{\min }$ must be at least twice the number of expected modes.

\section{AUTOMATED OUTPUT-ONLY MODAL IDENTIFICATION TECHNIQUE}

\subsection{Stabilization diagrams and spurious modes elimination}

The quality of stabilization diagrams can always be compromised by poor signal-to-noise ratio of the data. In addition, the choice of high model orders (often necessary for identifying weakly excited modes) or an inadequate selection of the algorithm parameters can also contribute to increase the computational time for the automatic interpretation of stabilization charts.

Therefore, three modal validation criteria are used in order to reject some spurious poles, cleaning up the stabilization diagram and consequently speeding up their automatic interpretation. First, all the identified poles that have a damping ratio less than 0 or higher than $10 \%$ are discarded. Of course, the choice to include damping values up to $10 \%$ is highly conservative for most of the applications to civil infrastructures. Subsequently, since in modal testing the identified structural mode shapes are generally complex vectors, further two wellknown modal validation criteria are used to evaluate the degree of complexity of the estimated mode shapes related to the identified poles: Mean Phase Deviation (MPD) and Modal Phase Collinearity (MPC) [8,22]. The MPD is a statistical indicator that measures the phase scatter (in degree) of each identified modal vector and its value tends to 0 for real normal mode shapes:

$$
M P D_{r}=\sqrt{\frac{\sum_{k=1}^{N}\left|\phi_{k r}\right|\left(\alpha_{k r}-M P_{r}\right)^{2}}{\sum_{k=1}^{N}\left|\phi_{k r}\right|}} \quad M P_{r}=\frac{\sum_{k=1}^{N}\left|\phi_{k r}\right| \alpha_{k r}}{\sum_{k=1}^{N}\left|\phi_{k r}\right|}
$$

in which $\phi_{k r}$ is the $k$-th element of the $r$-th mode shape, $\alpha_{k r}$ is its phase angle, $N$ indicates the DOF and $\mathrm{MP}_{\mathrm{r}}$ is the mean phase of the $r$-th mode shape. The MPC is a correlation index that evaluates the linear relation between the real (Re) and imaginary ( $\mathrm{Im})$ part of the identified modal vector $\phi_{r}$. If the imaginary part is strongly correlated to the real part, the MPC value is close to 1 indicating a mono-phase behaviour that is usually related to real normal modes:

$$
\begin{gathered}
M P C_{r}=\frac{\left\|\operatorname{Re}\left(\widetilde{\boldsymbol{\phi}}_{r}\right)\right\|^{2}+\left[\operatorname{Re}\left(\widetilde{\boldsymbol{\phi}}_{r}^{T}\right) \operatorname{Im}\left(\widetilde{\boldsymbol{\phi}}_{r}\right)\right]\left[2\left(\varepsilon_{r}^{2}+1\right) \sin ^{2}\left(\alpha_{r}\right)-1\right] \varepsilon_{r}^{-1}}{\left\|\operatorname{Re}\left(\widetilde{\boldsymbol{\phi}}_{r}\right)\right\|^{2}+\left\|\operatorname{Im}\left(\widetilde{\boldsymbol{\phi}}_{r}\right)\right\|^{2}} \\
\widetilde{\phi}_{k r}=\phi_{k r}-\frac{\sum_{k=1}^{N} \phi_{k r}}{N} ; \quad \varepsilon_{r}=\frac{\left\|\operatorname{Im}\left(\widetilde{\boldsymbol{\phi}}_{r}\right)\right\|^{2}-\left\|\operatorname{Re}\left(\widetilde{\boldsymbol{\phi}}_{r}\right)\right\|^{2}}{2\left[\operatorname{Re}\left(\widetilde{\boldsymbol{\phi}}_{r}^{T}\right) \operatorname{Im}\left(\widetilde{\phi}_{r}\right)\right]} ; \alpha_{r}=\arctan \left(\left|\varepsilon_{r}\right|+\operatorname{sign}\left(\varepsilon_{r}\right) \sqrt{1+\varepsilon_{r}^{2}}\right.
\end{gathered}
$$

To the best of the authors knowledge, so far there is no universal accepted index to assess the modal complexity of identified mode shapes from ambient vibration testing. Hence, the 
information provided by both indices is averaged together leading to one final index:

$$
M C I_{r}=\frac{1-M P C_{r}+\left(\frac{M P D_{r}}{45^{\circ}}\right)}{2}
$$

here denoted as Modal Complexity Index (MCI) and scaled between 0-1, with 0 indicating a mono-phase behaviour of the identified mode shape. The MCI has the only purpose to take profit of both estimated MPD and MPC values. On account of that, Fig. 4 shows how the MPC and the normalized MPD values slightly deviate from each other. In fact, a full consistency between both indices would provide a straight line with a slope equal to unity.

Anyhow, setting a threshold value of MCI deserves some remarks on the origin of complex modes. As well described in [23], the mode complexity depends on various factors, such as measurement errors (leakage, synchronization problems), identification issues (weaklyexcited modes, noise) and non-linear behavior of the structure (especially related to hysteretic or generally non-proportional damping). Therefore, assuming good test conditions, the choice of the threshold is highly related to the expected characteristics of the investigated structure.

A good operating way is to plot the frequencies of the identified poles versus the MCI (Fig. $5 \mathrm{a}-\mathrm{b}$ ) and choose a conservative value, in order to preserve the poles that seem clustered together and to reject the most relevant spurious poles. In the current application a threshold of 0.7 for the vertical modes (Fig. 5a) and 0.5 for the transversal modes (Fig. 5b) were assumed. Such high values are due to the complex behaviour of the bridge under study. Conversely, for a structure that is supposed to exhibit a clear linear behaviour lower thresholds between $0.3-0.4$ can be safely selected.

Moreover, introducing a modal complexity check and setting a static threshold allows to detect possible modal complexity variation that can be viewed as an additional warning index. In fact, as mentioned above, modal complexity variation can be linked either to changes of the structural response or to a possible malfunctioning of the monitoring system (e.g. loss of synchronized data acquisition).

\subsection{Automatic selection of structural modes}

After the elimination of the most relevant spurious poles, there is still the need to assemble together the structural modes that share the same modal properties. In this framework, several alternative procedures have been proposed during the last decade which can be mainly distinguished in hierarchical and non-hierarchical clustering methods.

Hierarchical clustering starts by considering each pole as a single cluster and computes the mutual distance or similarity between each cluster. In the subsequent step, the two closest clusters are merged together in one single cluster and the measure of similarity between the remained ones is performed again. As suggested by its own name, the clustering algorithm keeps going on forming clusters in a hierarchical fashion - in fact the number of clusters at each stage is one less than the previous one - until the mutual distance between the clusters exceeds a pre-defined threshold, as shown in [7]. A further application of a hierarchical algorithm was presented in [8] where the threshold to cut-off the hierarchical construction of clusters is not a user defined parameter, but is automatically set by taking advantage of the information obtained from a previous partitioning method that separates possibly physical and 
spurious modes.

On the other hand, non-hierarchical methods such as partitioning methods or k-means clustering assumes that the number of clusters of the expected modes are known in advance. However, in modal testing this is not often the case. In [17] such problem has been overcome by over-estimating the number of clusters and then applying a k-means clustering method. Subsequently, the first obtained clusters have been used to train a self-learning classification algorithm in order to set the thresholds that defines to which cluster an identified pole belongs. Once the thresholds are set, a final clustering is performed again. In that study the measure of similarity between the clusters includes the natural frequencies and damping ratio distance, introducing a modal vector correlation in a second stage in order to distinguish between closely spaced modes. Recently, a further non-hierarchical approach was presented in [9] consisting in a simple sequential procedure that starts to assume the first pole as an initial cluster. Subsequently a second pole is selected and the mutual distance between the initial cluster and the selected pole is computed. If such distance is lower than a pre-defined threshold the pole will be merged with the initial cluster, otherwise it will create a new second cluster. The procedure keeps going on until all the poles are gathered into a final number of clusters. A significant feature of the procedure presented in [9] relies on its extension in order to simultaneously consider variation of the input parameter of the modal identification algorithm.

Within this framework, the proposed clustering methodology tries to merge the different benefits of the above mentioned clustering approaches and, concurrently, to overcome some of their limitations.

The strategy of the adopted procedure consists of the following steps (see also Fig. 6):

1) starting from the system poles matrix, take the first available identified pole belonging to the lowest model order $n=n_{\min }$, and save the associated natural frequency, damping ratio and mode shape in the first row $j=1$ of the respective columns vectors $F_{k}$ and $D_{k}$ and matrix $M_{k}$ (with as many $m$ rows as the number of adopted model orders $n_{\max }-n_{\min }+1$ ); there will be as many $k$ sets of this matrices/vectors as the final number of clustered structural modes;

2) compute the mean value $f_{a v}$ and the mean modal vector $\phi_{a v}$ of the non-zero elements contained in vector $F_{k}$ and in matrix $M_{k}$, and calculate the distances, $d_{n, i}$, in terms of frequency and Modal Assurance Criterion (MAC) variation between the previous defined mean values and all frequencies and modal vectors $i$ belonging to the successive model order $n=n+1[6,7,9]$ :

$$
d_{n, i}=\left(d_{n, i}^{f}\right)+\left(d_{n, i}^{M A C}\right)=\left(\frac{\left|f_{a v}-f_{n, i}\right|}{f_{a v}}\right)+\left(1-\frac{\left|\phi_{a v}^{*} \phi_{n, i}\right|^{2}}{\left(\phi_{a v}^{*} \phi_{a v}\right)\left(\phi_{n, i}^{*} \phi_{n, i}\right)}\right)
$$

3) keep in selection the poles that produce a distance $d_{n, i}$ lower than an automatically defined threshold $d_{\max }$ (which is explained later). If more than one pole is detected, select the pole with the minimum distance $d_{n, i}$ and save its modal parameters in the row $j=j+1$ of the vectors and matrix constructed in step1. If no mode is detected, put 0 ;

4) repeat step 2 and 3 in a sequential manner, first defining the references $f_{a v}$ and $\phi_{a v}$, and then computing the distances taking into account the poles belonging to the successive model 
order $n=n+1$;

5) once achieved the highest model order $n_{\max }$, if the number of modes contained in the frequency vector $F_{k}$ are higher than a third of the vector size $m$, a group of consistent structural modes is definitely identified, otherwise the vector will be deleted;

6) turn to step 1, select the next available identified pole (that not belongs to the previous defined group) and construct the two row vectors and the modal vector matrix again; the procedure continues until all available poles have been scanned.

Once $k$ groups of consistent structural modes are defined, the most representative values of modal properties from each group are extracted. Frequency, mode shape, MPC and MPD are obtained averaging the respective values which belong to the same cluster. Conversely, the most representative damping value for each mode is evaluated by calculating the median of all single damping estimation contained in the respective cluster. Since the damping estimation is often scattered, the median has the advantage of weighing less the outliers. Finally, for each cluster the standard deviations of all modal properties are extracted.

The threshold $d_{\max }$ that drives the decision process of clustering is a self-adaptable parameter obtained in the following way:

1) a starting value of $d_{\max }$ needs to be a priori defined. Such value always depends on how the distance between two poles is computed; therefore considering equation (5) values of $d_{\max }$ between 0.02 and 0.04 are good starting points, their influence on the final results is low;

2 ) if a cluster $k$ starts to contain more than two poles, the square root of the median absolute deviation (6) of the distances $d_{n, i}$, computed so far between the gathered poles and the references $f_{a v}$ and $\phi_{a v}$, is calculated:

$$
d_{\max }=\sqrt{M A D}=\sqrt{\operatorname{median}\left(d_{n, i}-\operatorname{median}\left(d_{n, i}\right)\right)}
$$

3) once calculated, such value is used as the new threshold and is iteratively updated until the first cluster is completed. When a new cluster starts, the pre-defined starting value is used again as a threshold while the gathered poles are lower than 3 . If the new cluster keeps growing up, $d_{\max }$ is computed again by equation (6) using the distances calculated so far for the new cluster.

In such way, the threshold is iteratively self-trained and updated basing on the data collected in the considered cluster. This is a significant advantage compared to the methods used in [7,9] that rely on static thresholds. In fact, Table 1 clearly shows that each cluster can contain different variations of frequency: for example, the standard deviation of the gathered frequencies of mode V-2 and V-9 exhibit a significant difference in order of magnitude. The same behaviour can be observed on the transversal modes, like mode H-1 and H-6. In addition, during a long-term dynamic monitoring - although a well-tuned static threshold based on some initial data-set might be used - there is no guarantee that a user-defined static parameter will keep being appropriate for future recorded data-set, which might have a different signal-to-noise ratio. In this context, an automated self-adaptable threshold seems to better fit the requirements of a dynamic monitoring system. As mentioned before, previous works $[8,17]$ already remarked this aspect and proposed different solutions. Both approaches have been successfully applied on different case studies: an airplane flight flutter test in [17] and a post-tensioned concrete bridge in [8]. However, both procedures are more 
computationally demanding. For example the one in [17] needs to perform a first clustering to properly train the threshold through a support vector machine algorithm; once the threshold is set, a final clustering is performed again. On the other hand, the methodology proposed in [8] is based on a previous partitioning method that performs a first separation between physical poles and spurious ones. This first step also allows to train the threshold that is then used in a subsequent hierarchical clustering method, which is computationally demanding by its own. In fact, hierarchical clustering computes the whole similarity matrix between all the identified poles and clusters after each clustering iteration. On the contrary, the main advantage of the proposed procedure lies in its simple implementation and fast computation: the clustering process requires less iterations, at the same time, the threshold is trained and updated during the clustering itself.

The choice of using the square root of the median absolute deviation to calculate the $d_{\max }$ was mainly driven by two reasons: a) the MAD is a more robust measure of data dispersion, being less sensitive to outliers if compared to the classical standard deviation [24]; b) the square root simply allows to increase the computed MAD by an order of magnitude (its values are always lower than unity) in order to obtain a conservative threshold based on the data dispersion for the clustering process.

It is worth to include two further comments about the above described procedure:

(a)the introduction of the averaging process - that defines for each scan of poles the reference values $\left(f_{a v}\right.$ and $\left.\phi_{a v}\right)$ - has been adopted in order to preserve the information of the previous assembled stable modes within the cluster and to avoid any specific dependence of the clustering method to one particular clustered pole;

(b)some readers might wonder why the similarity measure in (5) does not include the damping ratio parameter, as for example suggested in [17]; the reason simply lies on the high scattering of damping ratios observed for some modes, for example in Fig. 7e the vertical modes at $2.4 \mathrm{~Hz}, 7.4 \mathrm{~Hz}$ and $11 \mathrm{~Hz}$ or in Fig. $7 \mathrm{f}$ the transversal modes at $2.0 \mathrm{~Hz}$, $2.5 \mathrm{~Hz}, 3.1 \mathrm{~Hz}$ exhibit a higher and different dispersion of damping ratios than the one observed for the other modes; on the other hand, the MAC index in (5) is fundamental since it differentiate modes with closely spaced frequencies.

Finally, Figs. 7a,c and Figs. 7b,d highlight the performance of the clustering process applied on a recorded data set from the monitoring system of the San Michele Bridge, showing clear clustered vertical and transversal modes, respectively.

Table 1 also reports some further statistic information regarding the averaged MAC value after each clustering iteration for each mode, the mean MCI (4) index and the final threshold $d_{\max }(6)$ computed for each clustered mode, vertical and transversal ones. It should be noticed that the order of magnitude of $d_{\max }$ is effectively different for some modes, supporting the fact that static thresholds might be inappropriate for modal clustering applied to a permanent dynamic monitoring context.

\subsection{Mode tracking for continuous dynamic monitoring}

In the context of permanent ambient vibration monitoring, an important issue is related to the choice of the most meaningful modes of the structure and to how this modes evolve or might change in time. To do this, the modes identified from each recorded data-set need to be 
compared and linked to those which define the starting baseline list. Such process is usually called modal tracking [8]. However, especially for complex structures, the choice of a baseline list of structural modes is not always straightforward.

The common practice is to define the baseline modal parameters through previous ambient vibration tests, considered mandatory to provide prior knowledge of the dynamic characteristics of the investigated structure. Usually, the structural modes that are clearly identifiable and exhibiting a reasonable physical mode shape are included in the baseline list. However, especially for complex structures, it is not always possible to identify clearly the most meaningful modes. Therefore, a better approach consists in performing a preliminary one-day monitoring test, also useful to optimize the number of points to be instrumented. The information collected during the one-day test is essential to better identify the modes and to understand their behavior under different environmental and operational conditions.

Once defined a baseline list, it becomes necessary to develop a procedure in order to track the evolution in time for the selected structural modes. Despite modal tracking is a wellknown problem in ambient vibration monitoring, only few works in the literature devoted some observations on this aspect. Practically, the problem might be simply solved by applying a $k$-mean clustering algorithm, in which $k$ stands for the number of structural modes defined in the baseline list. Therefore, in order to link the continuously identified modes to the reference ones a similarity index between modes can be used. For example in [7], the MAC index and the variation of frequencies have been successfully adopted. For both parameters a static threshold was fixed: an identified mode is linked to the reference one only if the MAC results higher than 0.8 and their frequency variation is lower or equal to $15 \%$.

A further static threshold-based modal tracking strategy is also proposed in [15], where an automated FDD procedure joins together the modal identification and tracking process. The whole methodology relies on prior definition of reference mode shapes and detection of modal domains for each subsequent dataset, by performing comparison in terms of MAC between the reference mode shapes and the estimated singular vectors at each frequency line. In this case, if a certain number of singular vectors exhibit a MAC value higher than a predefined threshold, a modal domain is identified and linked to the reference mode. However, since natural frequencies of the modes may change due to the variation in the environmental and operational conditions, it is often necessary to manually update such thresholds, otherwise the tracking procedure might fail, affecting any subsequent SHM strategy. In order to avoid such problem, relatively high threshold values might be used, but such solution can fail if the current dataset is characterized by closely spaced modes, which exhibit some degree of coupling between their mode shapes. In [25] a further FDD-based procedure was presented and an alternative solution of modal tracking is proposed by using an adaptive MAC rejection level which is continuously set and updated at each iteration.

The tracking methodology herein proposed exploits the similarity measurement presented in [7], that computes the frequency variation and MAC index (necessary to distinguish any closely spaced frequencies) between the reference modes and the ones identified in subsequent data sets (Fig. 8). However, instead of using static rejection thresholds, the filtering and linking process is performed in an adaptive way, updating - independently for each structural mode - the MAC and frequency variation threshold after each new data set. In such way, the methodology keeps automatically track of any variation of modal parameters 
avoiding further user-interaction.

In the following, each single step of the developed procedure is described (see also Fig. 8):

1) for each reference mode in the baseline list, compute the frequency and MAC variation with all the identified modes in the new recorded data set $h=1$;

2) then, for each reference mode keep the modes identified in data set 1 that induce $a d_{h, i}^{f}$ and a d ${ }^{\mathrm{MAC}}{ }_{\mathrm{h}, \mathrm{i}}$ lower than two self-adaptable thresholds $\left(\mathrm{d}_{\mathrm{i}, \max }^{\mathrm{f}}\right.$ and $\mathrm{d}_{\mathrm{i} \text {,min }}^{\mathrm{MAC}}$ ) for each reference mode. Subsequently, select and group the mode which is closer to the related reference one;

3 ) in order to scan the consecutives data set, $h=h+1$, repeat step 1 and 2 keep using as reference modes the ones selected for the baseline list. An alternative approach may consist in iteratively updating the reference modes with the last identified ones, that allows to better follow and track the variation of each mode, however - for SHM purposes - keeping fixed the reference modes means fixing a reference structural health state in time, to which all the future structural states should be compared.

Similarly to what described in the previous section, before starting the updating process of the thresholds, two starting values need to be a prior fixed. In this case, we can safely assume $d_{i, \max }^{f}$ and $d_{i, \max }^{M A C}$ equal to $10 \%$ for each reference mode. Once the tracking process starts to group together more than 2 consecutive identified modes for each reference mode, equation (7) sets a pair of thresholds for each mode. Equation (7) is applied to the distances in terms of frequency $\left(d_{h, i}^{f}\right)$ and MAC $\left(d_{h, i}^{M A C}\right)$ computed so far between the tracked modes and the reference ones:

$$
\begin{aligned}
& d_{i, \max }^{f}=\sqrt{s t d\left(d_{h, i}^{f}\right)\left(1+\frac{1}{\log (h)}\right)} \\
& d_{i, \max }^{M A C}=\sqrt{\operatorname{std}\left(d_{h, i}^{M A C}\right)\left(1+\frac{1}{\log (h)}\right)}
\end{aligned}
$$

where std is the standard deviation of all the computed $d_{h, i}^{f}$ and $d_{h A, i}^{M A}$, in which $i$ is the index of the reference mode and $h$ indicates the number of the data set.

Both values are used as new thresholds and are iteratively updated until the end of the monitoring. The reason of using the logarithmic factor is to weight the prediction interval in relation to the number $(h)$ of the so far computed distances (for each mode $i$ ). The procedure allows to build tailored prediction levels for each mode (avoiding possible tracking errors in presence of closely spaced modes, as mentioned before). Concurrently, the whole tracking procedure gets capable to follow the evolution of modal parameters due to operational and/or environmental condition. Of course, there is no guarantee that such dynamic thresholds are able to cover the effects induced by extreme environmental conditions or by damage. In such case, the modal tracking process might simply fail (i.e. significant decreasing of the tracking rate compared to a previuos period) or the computed thresholds (if able to cover the previous mentioned effects) might start to grow up and will eventually exceed a priori fixed warning level (e.g. $15 \%$ for $d_{i, \max }^{f}$ and $30 \%$ for $d_{i, \max }^{M A C}$ ). However, both cases can be viewed as a sort of warning index, suggesting that a user interaction can no longer be postponed. 


\section{APPLICATION ON THE SAN MICHELE BRIDGE}

\subsection{Identification and tracking of the vertical and transversal modes}

The two starting baseline lists of the vertical and horizontal modal parameters are reported in Fig. 9 and Fig. 10, respectively.

Fig. 9 highlights that the first modes are dominant bending modes, whereas mode V-11 is characterized by torsion behaviour.

The horizontal modes (Fig. 10) turned out to have a more regular behaviour than the vertical ones. As also highlighted in Fig. 7d, the transversal dynamic response is characterized by a large number (15) of clear and well-separated modal frequencies up to $5 \mathrm{~Hz}$.

Once defined the baseline list, the identification and tracking procedure was performed adopting the input parameters summarized in Table 2.

The results of the automated identification and tracking procedure of the natural frequencies, based on the SSI-Cov method, are shown in Fig. 11 and Fig. 12, for the vertical and horizontal modes respectively.

Table 3 reports the statistical information of the obtained results, in terms of average frequency, $f_{\text {mean }}$, standard deviation and success rate of identification and tracking. For validation purpose, some of the vertical modes were identified and tracked by a manual inspection of 723 stabilization diagrams produced by the Data-driven SSI technique available in the commercial software ARTeMIS [26]. This is a quite unique set of results that permit a true evaluation of the automated algorithm.

A further validation is presented in Fig. 13 and Fig. 14, that represent a top view of the first singular values (SV) of the spectral matrix [16] calculated for each data set with a frequency resolution of $0.0195 \mathrm{~Hz}$. The hotter colors represent the frequencies with a higher energy content and help visualizing the time evolution of the more excited natural frequencies.

\subsection{Discussion of the results}

A first inspection of Figs. 11-12 reveals a very good and robust performance of the automated procedure. All the expected modes are clearly identified exhibiting a fairly good identification rate, as also shown in Table 3. It should be noted that between 18 to 31 March 2012 some data files are missing, due to issues related to the powering of the monitoring system. Furthermore, a more detailed inspection of one of the modes (i.e. mode V-7), points out a cyclic evolution of the tracked natural frequencies (Fig. 15). In fact, just by overlaying the temperature variation recorded by the two thermocouples, it clearly appears that the fluctuation of the identified frequencies is strongly driven by thermal effects. Hence, by extending such kind of inspection to each single mode, it comes out that most of the frequency variation can be explained by environmental and/or operational factors (i.e. temperature and traffic-induced acceleration). However, the study of the related correlations does not fall within the scope of the current work, more details are provided in [27].

The comparison of the SV-based horizontal alignments shown in Figs. 13-14, with the natural frequencies identified by the SSI-Cov based procedure (Figs. 11-12), provides an excellent agreement. Furthermore, some observations can be outlined:

(a)the vertical modes at 2.5, 7.4 and $8.6 \mathrm{~Hz}$ and the transversal modes at 2, 2.2, 2.5 and $3.2 \mathrm{~Hz}$ seem to be ill-exctitated and/or with a discontinuous excitation that seems to be higher 
during some hours (most-likely during those hours characterized by a higher presence of traffic). Despite this, the adopted procedure allowed a fairly good identification of the frequencies of those modes;

(b)the vertical modes at 5.2, 7.4, 11,11.8 and $12.05 \mathrm{~Hz}$ and the transversal modes at 4.07 and 4.14 occasionally split into two closely spaced frequencies (Figs. 16a-b). Concerning the vertical ones, such closely spaced modes are also characterized by a high degree of coupling between the mode shapes. Nevertheless, the choice of self-adaptable rejection levels for the clustering and tracking process and of the input parameters reported in Table 2 allows to overcome such possible problem, providing an excellent performance of the automated algorithm.

Figs. 17a-b shows the advantage of using dynamic thresholds. For each mode, the last updated MAC and frequency threshold, according to equation (7), was plotted. It clearly demonstrates how each mode deserves a different and tailored MAC rejection level. On the contrary, the frequency variation for each mode during the tracking process seems to be more similar to each other, at least in the used data-sets.

As mentioned before, for further validation purposes the estimates of some vertical modes obtained by the proposed procedure are compared to those identified by a manual inspection of 723 stabilization diagrams, using the SSI-Data technique implemented in the commercial software ARTeMIS. The results obtained by applying the present automated procedure and the manual SSI-data technique are summarized in Table 3 through some statistical indices. Table 3 shows that the standard deviations and mean values of the modes identified using the two procedures are almost similar, confirming the accuracy of the automated algorithm. On the other hand, a clear difference in terms of identification rate appears for the first vertical mode at $2.5 \mathrm{~Hz}$. Such difference is mainly due to two reasons: (a) a different selection of reference channels was done when the SSI-Data method has been used and (b) the differences existing between the SSI-Cov and the SSI-data algorithms [11].

It should also be mentioned that the SSI-Data was applied before the development of the present algorithm, in order to validate a previous and different automated procedure [21] capable of only ocassionaly identifying the vertical modes at $7.4 \mathrm{~Hz}, 8.6 \mathrm{~Hz}, 12.06$ and 12.5 Hz. Since a manual analysis is a time consuming process, the same set of identified modes was used in the present work.

\section{CONCLUSIONS}

An automated modal identification and tracking procedure, based on parametric methods that involve the construction of stabilization diagrams, has been presented and applied on data continuously collected during one month by the permanent dynamic monitoring system installed on the historic San Michele Bridge.

In detail, the proposed approach exploits the introduction of self-adaptable thresholds regarding the modal clustering and tracking process and its simple implementation, that guarantees less computational effort compared to already existent algorithms. Furthermore, the clustering and tracking algorithms provide for each modal cluster a different and independent iteratively updated threshold. This last property is a fundamental aspect that overcomes most of the problems connected to the selection of global static rejection levels, which ill-suit to the issues related to continuous dynamic monitoring for SHM purposes. 
At the same time, the described procedure was developed in the spirit of providing a good trade-off between automation and gaining confidence with the recorded data and obtained results. This latter aspect is crucial in SHM, since good quality and accurate results are fundamental to well interpret the dynamic behaviour of the structure. In fact, the existence of only few chosen tuning parameters can be considered as a good practice since it allows a better optimization of the algorithm for each application. For example, in the current case study a static threshold for the MCI needed to be tuned since it appeared to be a sensitive parameter for the structure under investigation. This allows the analyst to keep a higher control on that modal property.

In terms of validation, the most challenging results of the proposed methodology were compared to the ones independently obtained by a manual interpretation of a large number of stabilization diagrams. Concurrently, the whole results were also checked against color maps provided by a partial application of the FDD method. A very good performance of the proposed procedure clearly appears, demonstrating good results even for weakly excited modes and showing that the introduction of adaptive thresholds for both modal clustering and tracking provides a higher flexibility to the methodology, especially if compared to static thresholds based algorithms. In addition, all the expected modes are well identified and tracked, exhibiting a fairly good identification rate.

It is worth mentioning that the proposed methodology can still be improved with the inclusion of two further ingredients: (a) an automated and adaptive selection of the input parameters of the modal identification algorithm itself (an attempt was already presented in $[9,28]$ ), as the SSI methods are sensitive to the variation of those parameters; (b) the estimation of the uncertainty level for each identified mode, due to the modal identification $[29,30]$ and clustering process [9]. Nevertheless, the proposed methodology already seems highly promising for application in continuous dynamic monitoring systems for SHM purposes.

\section{REFERENCES}

[1] Brownjohn, J. M. W., Moyo, P., Omenzetter, P., Chakraborty, S. Lessons from monitoring the performance of highway bridges. Structural Control and Health Monitoring 2005; 12(3-4): 227-244.

[2] Koo, K. Y., Brownjohn, M. W., List, D. I., Cole, R. Structural health monitoring of the Tamar suspension bridge. Structural Control and Health Monitoring 2013; 20: 609-625.

[3] Cunha, Á., Caetano, E., Magalhães, F., Moutinho, C. Recent perspectives in dynamic testing and monitoring of bridges. Structural Control and Health Monitoring 2013; 20: 853-877.

[4] Ramos, L. F. , Marques, L., Lourenço, P. B., De Roeck, G., Campos-Costa, A., Roque, J. Monitoring historical masonry structures with operational modal analysis: two case studies. Mechanical Systems and Signal Processing 2010; 24: 1291-1305.

[5] Cabboi, A., Gentile, C., Saisi, A. Frequency tracking and F.E. model identification of a masonry tower. In Proceedings of the $5^{\text {th }}$ International Operational Modal Analysis Conferences, Guimarães, Portugal, 2013.

[6] Saisi, A., Gentile, C., Guidobaldi, M. Post-earthquake continuous dynamic monitoring of the Gabbia Tower in Mantua, Italy. Construction and Building Materials 2015; 81: 101-112.

[7] Magalhães, F., Cunha, Á., Caetano, E. Online automatic identification of the modal 
parameters of a long span arch bridge. Mechanical Systems and Signal Processing 2009; 23: 316-329.

[8] Reynders, E., Houbrechts, J., DeRoeck, G. Fully automated (operational) modal analysis. Mechanical Systems and Signal Processing 2012; 29: 228-250.

[9] Ubertini, F., Gentile, C., Materazzi, A.L. Automated modal identification in operational conditions and its application to bridges. Engineering Structures 2013; 46: 264-278.

[10] Van Overschee, P., De Moor, B. Subspace identification for linear systems: Theory, implementation, applications. Kluwer Academic Publishers, 1996.

[11] Peeters, B. System identification and damage detection in civil engineering structures. $\mathrm{PhD}$ Thesis, Katholieke Universiteit Leuven, Belgium, 2000.

[12] Magalhães, F., Reynders, E., Cunha, Á., De Roeck, G. Online automatic identification of modal parameters of a bridge using p-LSCF method. In Proceedings of the $3^{\text {rd }}$ International Operational Modal Analysis Conference, Portonovo, Italy, 2009.

[13] Peeters, B., Van Der Auweraer, H. PolyMax: a revolution in operational modal analysis. In Proceedings of the $1^{\text {st }}$ International Operational Modal Analysis Conference, Copenhagen, Denmark, 2005.

[14] Rainieri, C., Fabbrocino, G. Automated output-only dynamic identification of civil engineering structures. Mechanical Systems and Signal Processing 2010; 24: 678-695.

[15] Busatta, F., Gentile, C., Saisi, A. Structural health monitoring of a centenary iron arch bridge. In Proceedings of the $3^{\text {rd }}$ International Symposium on Life-Cycle Civil Engineering, Vienna, Austria, 2012.

[16] Brincker, R., Zhang, L., Andersen, P. Modal identification from ambient responses using frequency domain decomposition. In Proceedings of the $18^{\text {th }}$ International Modal Analysis Conference, San Antonio, USA, 2000.

[17] Goethals, I., Vanluyten, B., De Moor, B. Reliable spurious mode rejection using self learning algorithm. In Proceedings of ISMA 2004 International Conference on Noise and Vibration Engineering, Leuven, Belgium, 2004.

[18] Società Nazionale delle Officine di Savigliano. Il viadotto di Paderno sull'Adda (ferrovia Ponte S. Pietro-Seregno). Camilla e Bertolero, Torino, 1889.

[19] Nascè, V., Zorgno, A. M., Bertolini, C., Carbone, V. I., Pistone, G., Roccati, R. Il ponte di Paderno: storia e struttura. Restauro 1984; 13:73-4.

[20] Gentile, C., Saisi, A. Ambient vibration testing and condition assessment of the Paderno iron arch bridge (1889). Construction and Building Materials 2011; 25: 3709-3720.

[21] Busatta, F. Dynamic monitoring and automated modal analysis of large structures: methodological aspects and application to a historic iron bridge. $\mathrm{PhD}$ Thesis, Politecnico di Milano, Italy, 2012.

[22] Pappa, R. S., Elliott, K. B., Schenk, A. Consistent mode indicator for the eigensystem realization algorithm. Journal of Guidance, Control and Dynamics 1993: 16(5): 852858.

[23] Imregun, M., Ewins, D. J. Complex modes - origin and limits. In Proceedings of the $13^{\text {th }}$ International Modal Analysis Conference, Nashville, Tennessee, 1995.

[24] Mosteller, F., J. Tukey. Data Analysis and Regression. Upper Saddle River, NJ: Addison-Wesley, 1977.

[25] Rainieri, C., Fabbrocino, G., Cosenza, E. Near real-time tracking of dynamic properties for standalone structural health monitoring systems. Mechanical Systems and Signal Processing 2011; 25: 3010-3026.

[26] SVS (2012) ARTeMIS Extractor 2010 release 5.3, http://www.svibs.com.

[27] Cabboi, A., Gentile, C., Saisi, A. Vibration-based SHM of a centenary bridge: a comparative study between two different automated OMA techniques. In Proceedings of the $9^{\text {th }}$ International Conference on Structural Dynamics (EURODYN), Porto, 
Portugal, 2014.

[28] Pridham, B. A., Wilson, J. C. A reassessment of dynamic characteristics of the Quincy bay bridge using output-only identification techniques. Earthquake Engineering and Structural Dynamics 2005; 34: 787-805.

[29] Reynders, E., Pintelon, R., DeRoeck, G. Uncertainty bounds on modal parameters obtained from stochastic subspace identification. Mechanical Systems and Signal Processing 2008; 22: 948-969.

[30] Carden, P. E., Mita, A. Challenges in developing confidence intervals on modal parameters estimated for large civil infrastructure with stochastic subspace identification. Structural Control and Health Monitoring 2011; 18: 53-78. 
Table 1 : Statistics of the identified modal parameters of vertical (V, 27/02/2012,01:30) and transversal (H, 27/02/2012, 00:30) vibration modes after clustering.

\begin{tabular}{c|cc|cc|c|c|c}
\hline $\begin{array}{c}\text { Mode } \\
\mathrm{n}^{\circ}\end{array}$ & $\begin{array}{c}\text { Freq. } \\
(\mathrm{Hz})\end{array}$ & $\begin{array}{c}\text { st.dev } \\
(\mathrm{Hz})\end{array}$ & $\begin{array}{c}\text { Damping } \\
\text { ratio (\%) }\end{array}$ & $\begin{array}{c}\text { st.dev } \\
(\%)\end{array}$ & $\begin{array}{c}\text { mean } \\
\text { MAC }\end{array}$ & $\begin{array}{c}\text { MCI } \\
{[0-1]}\end{array}$ & $\mathrm{d}_{\max }$ \\
\hline \hline $\mathrm{H}-1$ & 0.9932 & $1.7 \mathrm{E}-05$ & 0.1386 & 0.0077 & 1.000 & 0.0085 & 0.0016 \\
$\mathrm{H}-2$ & 1.3483 & $3.5 \mathrm{E}-05$ & 0.3510 & 0.0060 & 1.000 & 0.0262 & 0.0041 \\
$\mathrm{H}-3$ & 1.6494 & $1.2 \mathrm{E}-04$ & 0.3863 & 0.0109 & 0.9997 & 0.0375 & 0.0147 \\
$\mathrm{H}-4$ & 2.0200 & 0.0011 & 0.2457 & 0.0574 & 0.9982 & 0.0637 & 0.0330 \\
$\mathrm{H}-5$ & 2.1771 & $5.8 \mathrm{E}-04$ & 0.4084 & 0.0125 & 0.9996 & 0.0840 & 0.0098 \\
$\mathrm{H}-6$ & 2.5246 & 0.0053 & 1.0729 & 0.3951 & 0.9959 & 0.2070 & 0.0534 \\
$\mathrm{~V}-1$ & 2.5614 & 0.0052 & 1.4014 & 0.8497 & 0.9978 & 0.0471 & 0.0359 \\
$\mathrm{H}-7$ & 2.8262 & $3.9 \mathrm{E}-04$ & 0.6185 & 0.0069 & 0.9997 & 0.0943 & 0.0158 \\
$\mathrm{H}-8$ & 3.1279 & $9.1 \mathrm{E}-04$ & 0.5186 & 0.0628 & 0.9968 & 0.1322 & 0.0312 \\
$\mathrm{~V}-2$ & 3.4197 & $4.2 \mathrm{E}-05$ & 0.1875 & 0.0059 & 1.0000 & 0.0201 & 0.0031 \\
$\mathrm{H}-9$ & 3.5829 & $5.5 \mathrm{E}-04$ & 0.5139 & 0.0093 & 0.9999 & 0.0549 & 0.0058 \\
$\mathrm{H}-10$ & 3.8182 & $8.9 \mathrm{E}-04$ & 0.5742 & 0.0112 & 0.9996 & 0.1162 & 0.0116 \\
$\mathrm{H}-11$ & 4.0756 & 0.0029 & 0.1772 & 0.0350 & 0.9990 & 0.0716 & 0.0228 \\
$\mathrm{H}-12$ & 4.1446 & $5.5 \mathrm{E}-05$ & 0.1744 & 0.0040 & 0.9998 & 0.1594 & 0.0066 \\
$\mathrm{H}-13$ & 4.3878 & $3.5 \mathrm{E}-04$ & 0.3991 & 0.0118 & 0.9992 & 0.1712 & 0.0209 \\
$\mathrm{~V}-3$ & 4.5019 & $1.1 \mathrm{E}-04$ & 0.5225 & 0.0054 & 1.0000 & 0.1956 & 0.0048 \\
$\mathrm{H}-14$ & 4.5659 & $3.5 \mathrm{E}-4$ & 0.3757 & 0.0129 & 0.9989 & 0.1162 & 0.0205 \\
$\mathrm{H}-15$ & 4.8095 & $8.1 \mathrm{E}-04$ & 0.3644 & 0.0153 & 0.9970 & 0.2021 & 0.0249 \\
$\mathrm{~V}-4$ & 5.2169 & $3.8 \mathrm{E}-04$ & 0.4861 & 0.0025 & 0.9998 & 0.0349 & 0.0042 \\
$\mathrm{~V}-5$ & 7.4117 & 0.0058 & 0.3681 & 0.1129 & 0.9981 & 0.0774 & 0.0219 \\
$\mathrm{~V}-6$ & 8.6884 & 0.0067 & 0.3128 & 0.0658 & 0.9986 & 0.2232 & 0.0262 \\
$\mathrm{~V}-7$ & 10.7397 & 0.0011 & 0.5418 & 0.0059 & 0.9990 & 0.2688 & 0.0224 \\
$\mathrm{~V}-8$ & 11.0472 & 0.0052 & 0.3141 & 0.0658 & 0.9939 & 0.2958 & 0.0592 \\
$\mathrm{~V}-9$ & 11.8613 & 0.0187 & 0.8579 & 0.0932 & 0.9982 & 0.3983 & 0.0253 \\
$\mathrm{~V}-10$ & 12.1073 & 0.0023 & 0.8223 & 0.0179 & 0.9808 & 0.4465 & 0.0650 \\
$\mathrm{~V}-11$ & 12.5612 & 0.0022 & 0.2344 & 0.0113 & 0.9916 & 0.2869 & 0.0424 \\
\hline
\end{tabular}

Table 2: Input parameters for the automatic modal identification and tracking procedures.

\begin{tabular}{|c|c|c|c|}
\hline \multicolumn{2}{|c|}{ Vertical modes (V) } & \multicolumn{2}{|c|}{ Transversal modes $(\mathrm{H})$} \\
\hline $\begin{array}{l}\text { SSI-Cov } \\
\text { algorithm }\end{array}$ & $\begin{array}{l}\text { noise mode } \\
\text { elimination }\end{array}$ & $\begin{array}{l}\text { SSI-Cov } \\
\text { algorithm }\end{array}$ & $\begin{array}{l}\text { noise mode } \\
\text { elimination }\end{array}$ \\
\hline 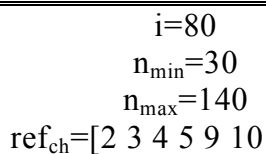 & $\begin{array}{c}\xi_{\max }=10 \% \\
M C I_{\max }=0.70\end{array}$ & $\begin{array}{c}\mathrm{i}=100 \\
\mathrm{n}_{\min }=50 \\
\mathrm{n}_{\max }=140 \\
\mathrm{ref}_{\mathrm{ch}}=\left[\begin{array}{llll}16 & 17 & 18 & 19\end{array}\right]\end{array}$ & $\begin{array}{c}\xi_{\max }=10 \% \\
M \mathrm{MCI}_{\max }=0.50\end{array}$ \\
\hline
\end{tabular}


Table 3 : Comparison of the statistical results between the manual analysis performed with SSI-Data/ARTeMIS and the automated modal identification procedure with SSI-Cov.

\begin{tabular}{|c|c|c|c|c|c|c|}
\hline \multirow[b]{2}{*}{$\begin{array}{l}\text { Mode } \\
\text { Id. }\end{array}$} & \multicolumn{3}{|c|}{$\begin{array}{c}\text { Automated procedure } \\
\text { (SSI-Cov) }\end{array}$} & \multicolumn{3}{|c|}{$\begin{array}{c}\text { Manual analysis } \\
\text { (SSI-Data/ARTeMIS) }\end{array}$} \\
\hline & $\begin{array}{l}f_{\text {mean }} \\
(\mathrm{Hz})\end{array}$ & $\begin{array}{c}\text { st.dev } \\
(\mathrm{Hz})\end{array}$ & $\begin{array}{l}\text { id. and } \\
\text { tracking } \\
\text { rate (\%) }\end{array}$ & $\begin{array}{l}\mathrm{f}_{\text {mean }} \\
(\mathrm{Hz})\end{array}$ & $\begin{array}{l}\text { st.dev. } \\
(\mathrm{Hz})\end{array}$ & $\begin{array}{l}\text { id. rate } \\
(\%)\end{array}$ \\
\hline "H-1 & 0.991 & $\overline{c 0.003}$ & 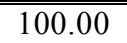 & - & - & - \\
\hline $\mathrm{H}-2$ & 1.345 & 0.004 & 100.00 & - & - & - \\
\hline $\mathrm{H}-3$ & 1.648 & 0.004 & 99.72 & - & - & - \\
\hline H-4 & 2.015 & 0.005 & 99.59 & - & - & - \\
\hline H-5 & 2.172 & 0.008 & 99.86 & - & - & - \\
\hline H-6 & 2.514 & 0.011 & 92.67 & - & - & - \\
\hline V-1 & 2.536 & 0.019 & 97.23 & 2.531 & 0.020 & 70.68 \\
\hline H-7 & 2.825 & 0.012 & 99.86 & - & - & - \\
\hline H-8 & 3.122 & 0.012 & 99.59 & - & - & - \\
\hline $\mathrm{V}-2$ & 3.415 & 0.008 & 99.72 & 3.414 & 0.009 & 99.72 \\
\hline H-9 & 3.573 & 0.016 & 100.00 & - & - & - \\
\hline $\mathrm{H}-10$ & 3.807 & 0.016 & 100.00 & - & - & - \\
\hline H-11 & 4.079 & 0.005 & 95.16 & - & - & - \\
\hline H-12 & 4.140 & 0.007 & 97.65 & - & - & - \\
\hline $\mathrm{H}-13$ & 4.381 & 0.012 & 99.72 & - & - & - \\
\hline V-3 & 4.513 & 0.009 & 100.00 & 4.521 & 0.012 & 97.37 \\
\hline H-14 & 4.560 & 0.014 & 99.86 & - & - & - \\
\hline H-15 & 4.804 & 0.010 & 98.89 & - & - & - \\
\hline V-4 & 5.212 & 0.008 & 99.86 & 5.210 & 0.008 & 99.86 \\
\hline V-5 & 7.408 & 0.016 & 97.51 & - & - & - \\
\hline V-6 & 8.668 & 0.020 & 88.80 & - & - & - \\
\hline V-7 & 10.732 & 0.015 & 99.86 & 10.737 & 0.016 & 96.40 \\
\hline V-8 & 11.012 & 0.020 & 99.45 & 11.020 & 0.022 & 89.21 \\
\hline V-9 & 11.862 & 0.032 & 99.45 & 11.861 & 0.022 & 94.05 \\
\hline V-10 & 12.062 & 0.032 & 97.37 & - & - & - \\
\hline V-11 & 12.520 & 0.030 & 84.65 & - & - & - \\
\hline
\end{tabular}




\section{CAPTIONS OF THE FIGURES}

Fig. 1 Views of the San Michele iron arch bridge (1889).

Fig. 2 Layout of the monitoring system.

Fig. 3 Effect of the time-lag $i$ on the modal properties of the first vertical mode $V-1$ (data sets 27/02/2012, 07:30 and 13:30).

Fig. 4 Consistency check between the MPC and MPD index before clustering: a) vertical modes (27/02/2012, 01:30); b) transversal vibration (27/02/2012, 00:30).

Fig. 5 Plot of natural frequencies of the identifies poles versus the MCI before clustering: a) vertical vibration modes (27/02/2012, 01:30); b) transversal vibration (27/02/2012, 00:30).

Fig. 6 Sketch of the adopted clustering procedure.

Fig. 7 Vertical modes data set 27/02/201, 01:30: a) stabilization diagram; c) stabilization diagram after clustering; e) damping ratio vs. frequency. Transversal modes data set 27/02/2012, 00:30: b) stabilization diagram; d) stabilization diagram after clustering; $f$ ) damping ratio vs. frequency.

Fig. 8 Sketch of the adopted mode tracking procedure.

Fig. 9 Baseline vertical modes identified from acceleration measurements with the SSI-Cov procedure (27/02/2012, 01:30).

Fig. 10 Baseline transversal modes identified from acceleration measurements with the SSI-Cov procedure (27/02/2012, 00:30).

Fig. 11 Identified natural frequencies from vertical acceleration measurements using the SSI-Cov application (27/02/2012-01/04/2012).

Fig. 12 Identified natural frequencies from horizontal acceleration measurements using the SSI-Cov application (27/02/2012-01/04/2012).

Fig. 13 First singular value plot of the vertical signals frequency content over time (27/02/201201/04/2012).

Fig. 14 First singular value plot of the horizontal signals frequency content over time (27/02/201201/04/2012).

Fig. 15 Cyclic behaviour of the identified vertical mode V-7 and temperature variation (upstream and downstream temperature sensors).

Fig. 16 Zoom of frequency splitting: a) vertical modes (data set 27/02/2012, 01:30); b) horizontal modes (data set 27/02/2012, 17:30).

Fig. 17 Adaptive modal tracking threshold for each mode obtained after the final set of data: a) vertical modes; b) horizontal modes. 


\section{Page 21 of 54}

Fig. 1
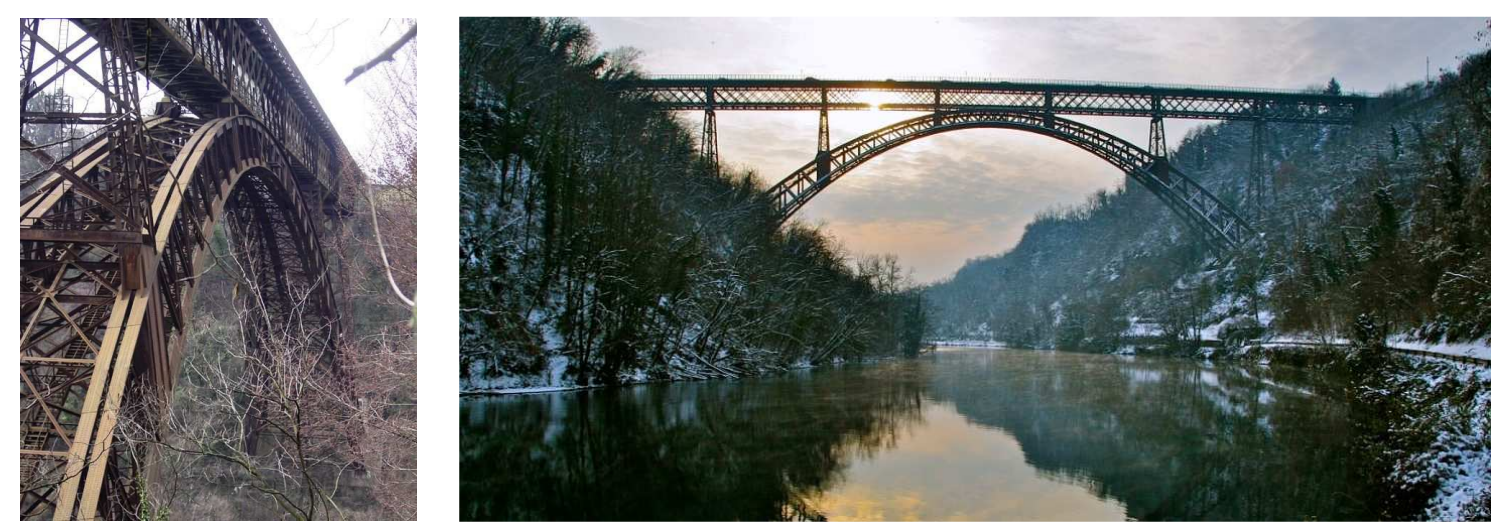


\section{Fig. 2}
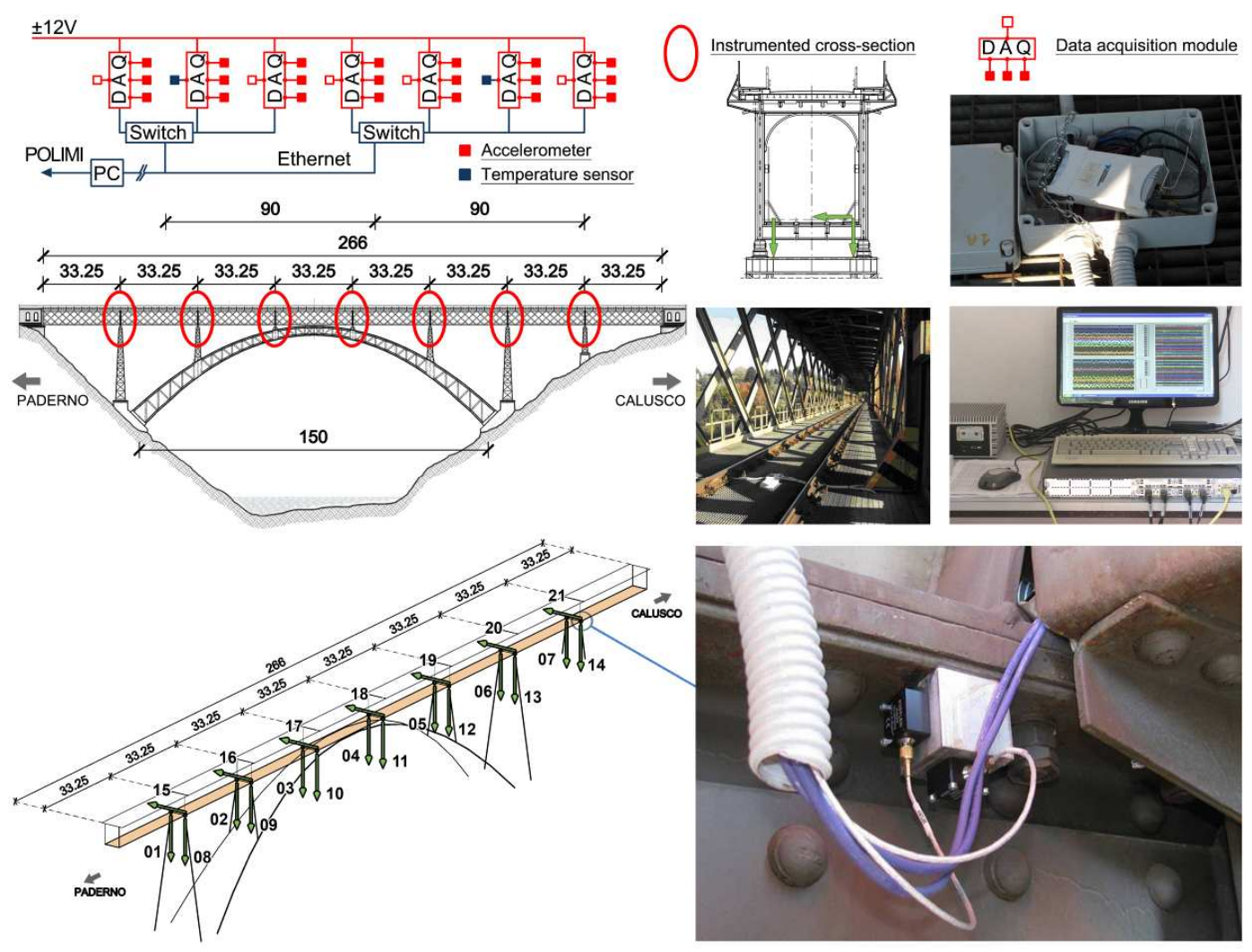

30

31

32

33

34

35

36

37

38

39

40

41

42

43

44

45

46

47

48

49

50

51

52

53

54

55

56 
1

2

3

4

5

6

7

8

9

10

11

12

13

14

15

16

17

18

19

20

21

22

23

24

25

26

27

28

29

30

31

32

33

34

35

36

37

38

39

40

41

42

43

44

45

46

47

48

49

50

51

52

53

54

55

56

57

58

59

60

Fig. 3

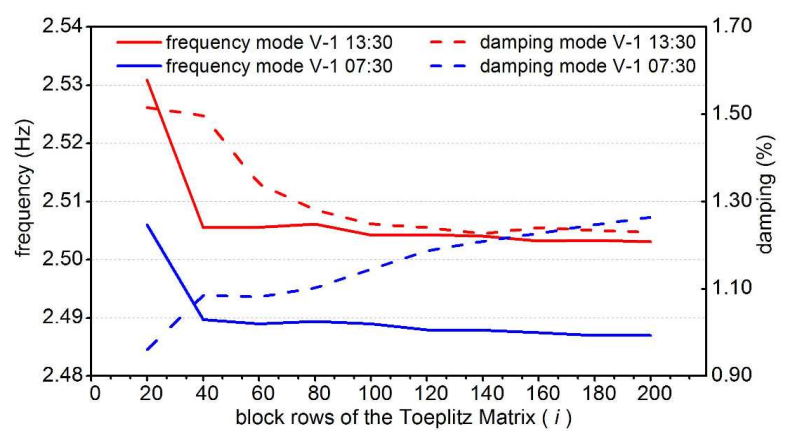


Fig. 4

a)

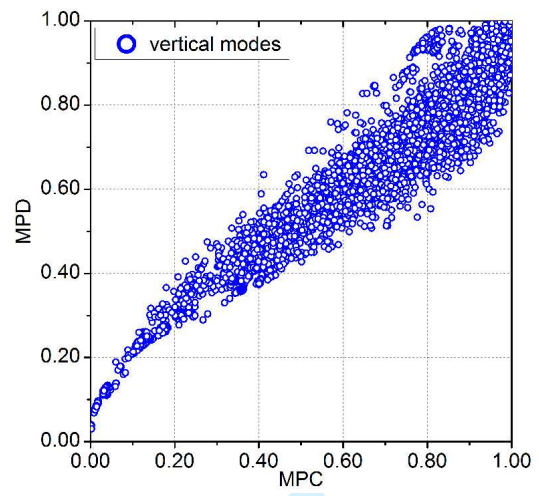

b)

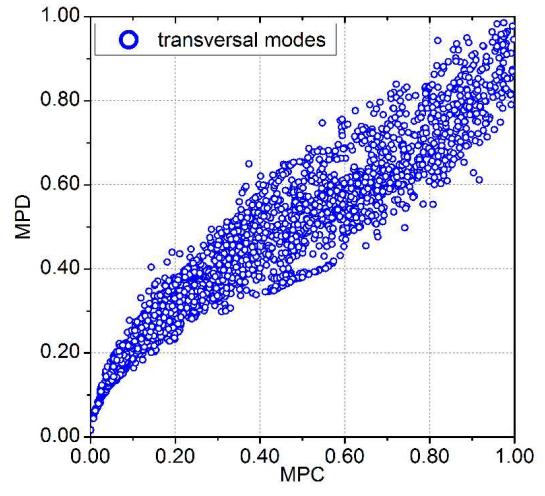


Fig. 5

a)

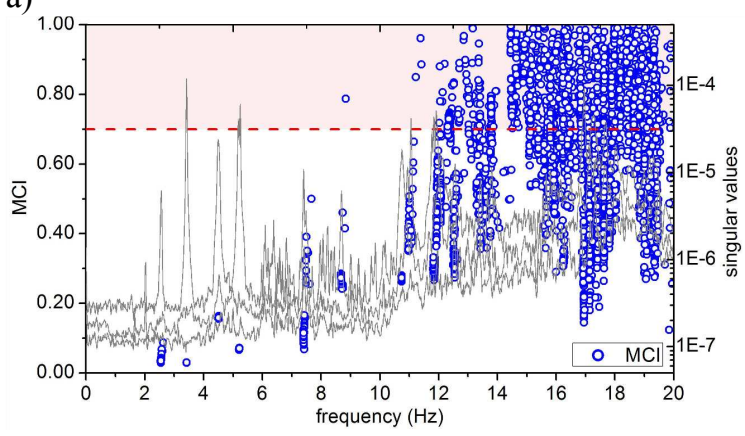

b)

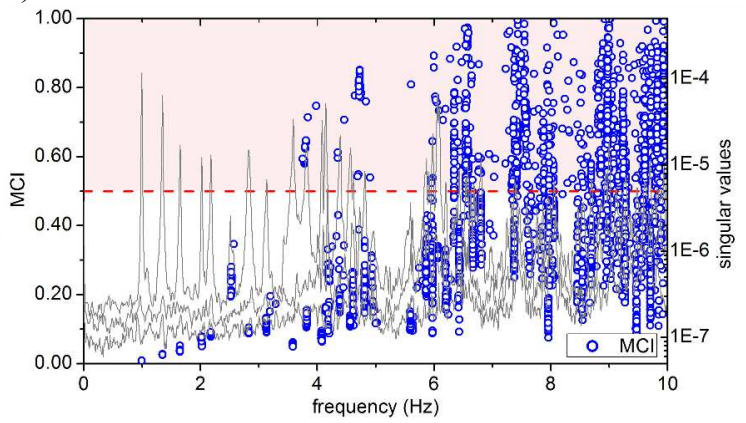

15

16

17

18

19

20

21

22

23

24

25

26

27

28

29

30

31

32

33

34

35

36

37

38

39

40

41

42

43

44

45

46

47

48

49

50

51

52

53

54

55

56

57

58

59

60 
Fig. 6

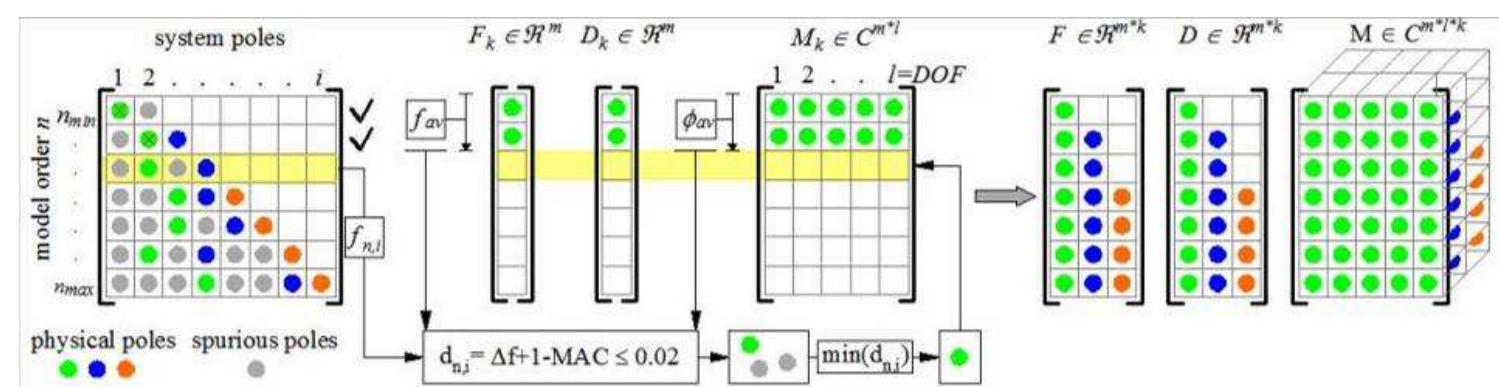


Fig. 7

a)

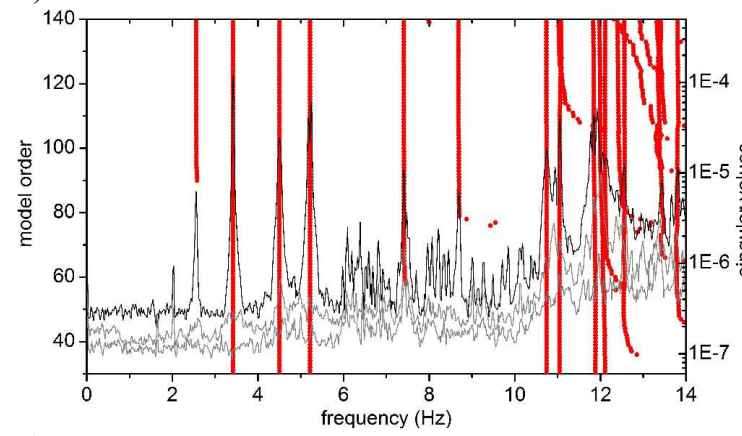

c)

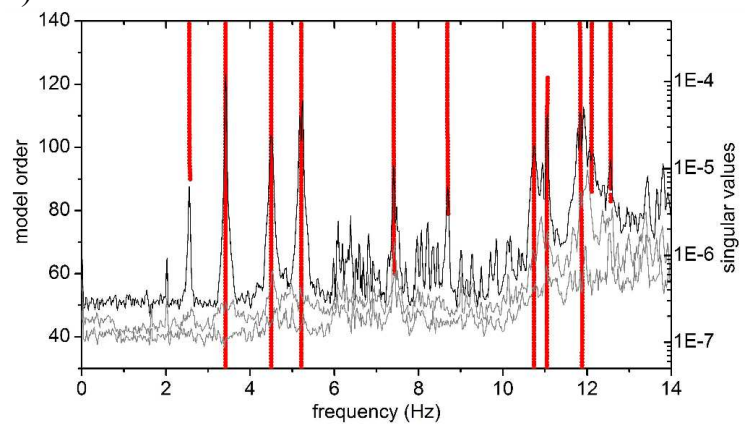

e)

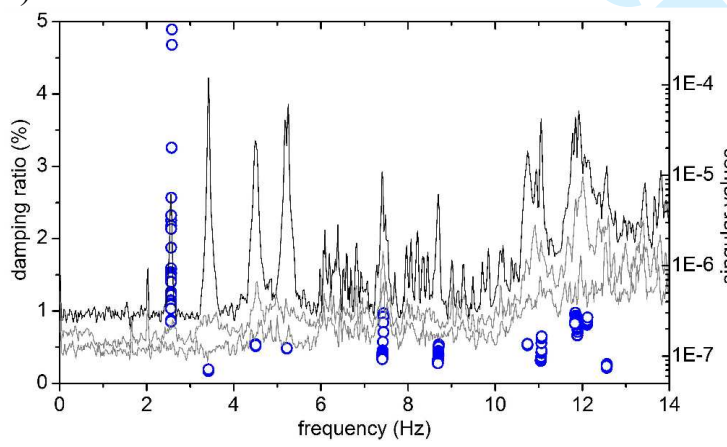

b)

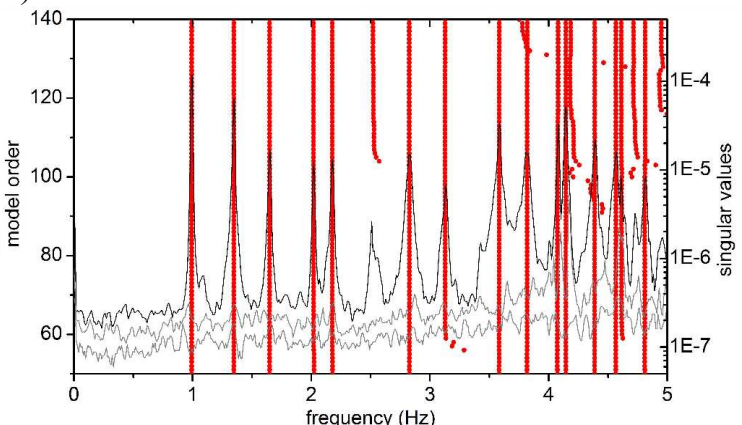

d)

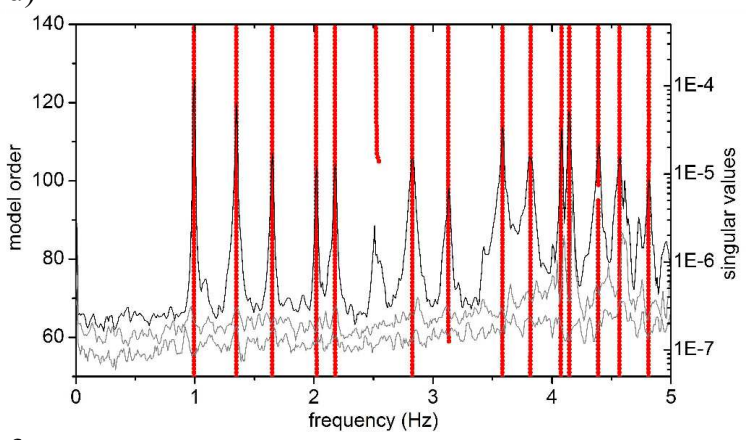

f)

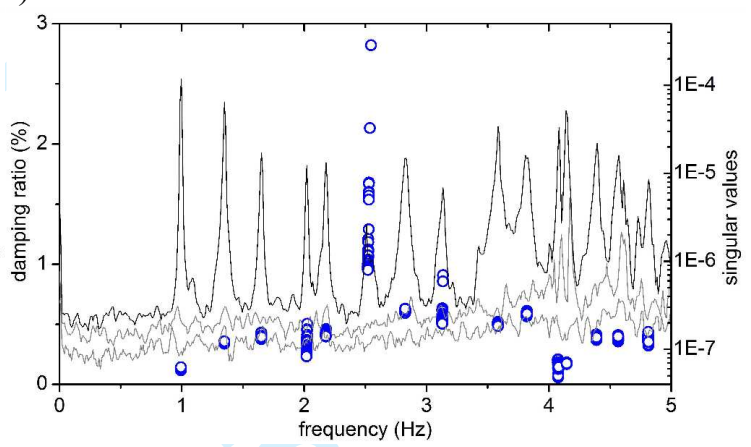


Fig. 8

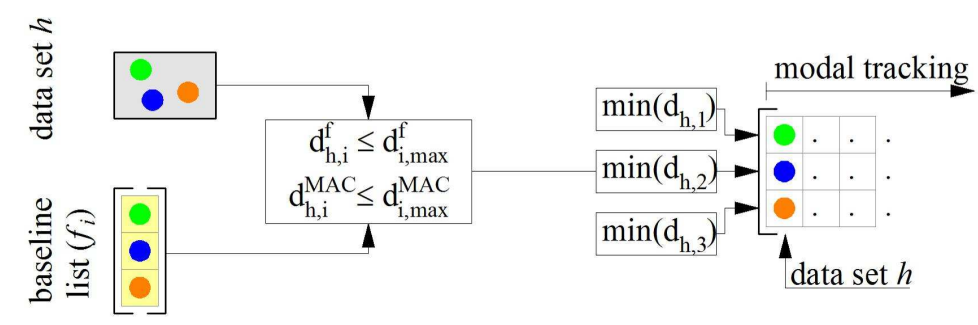


Fig. 9
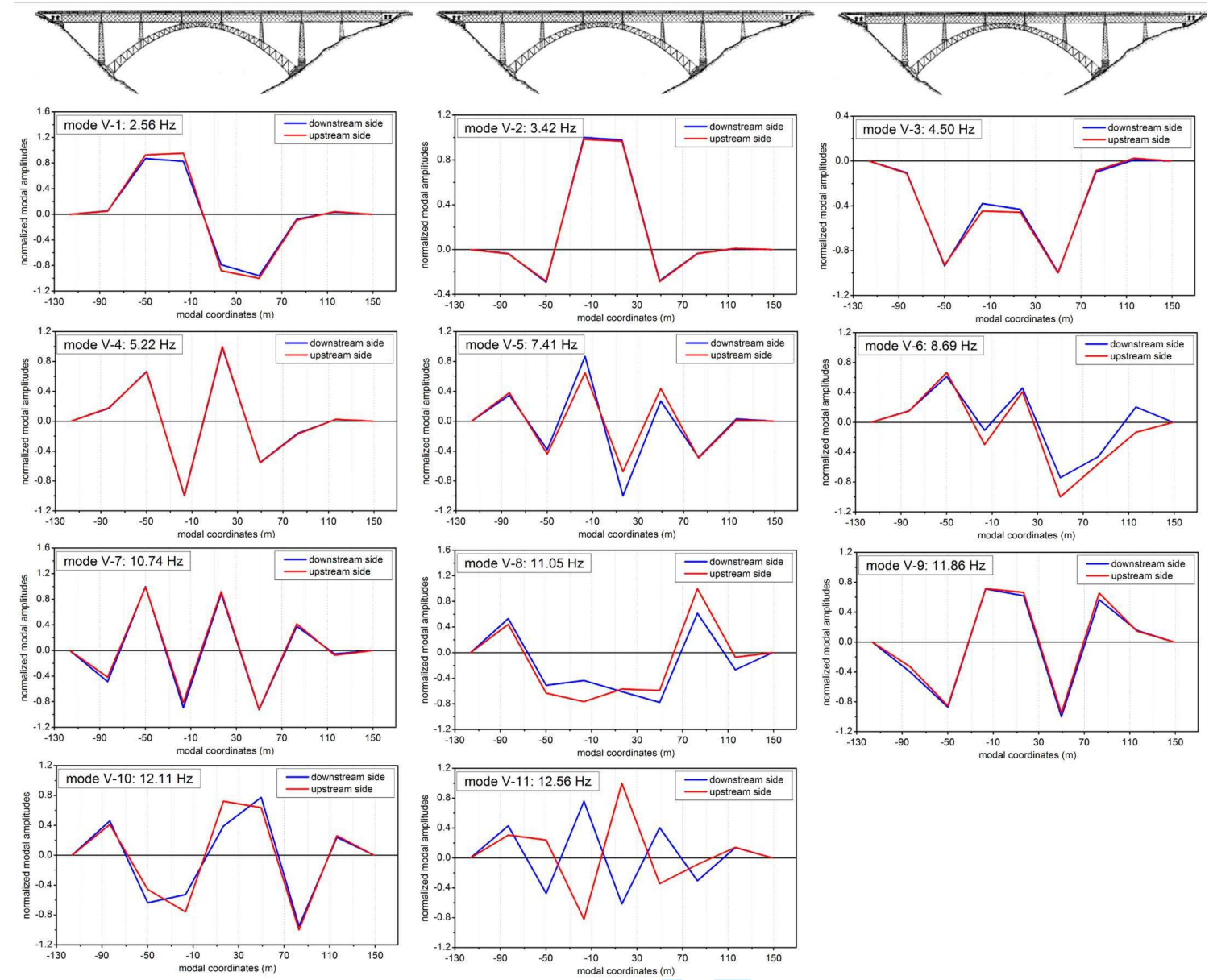


\section{Fig. 10}
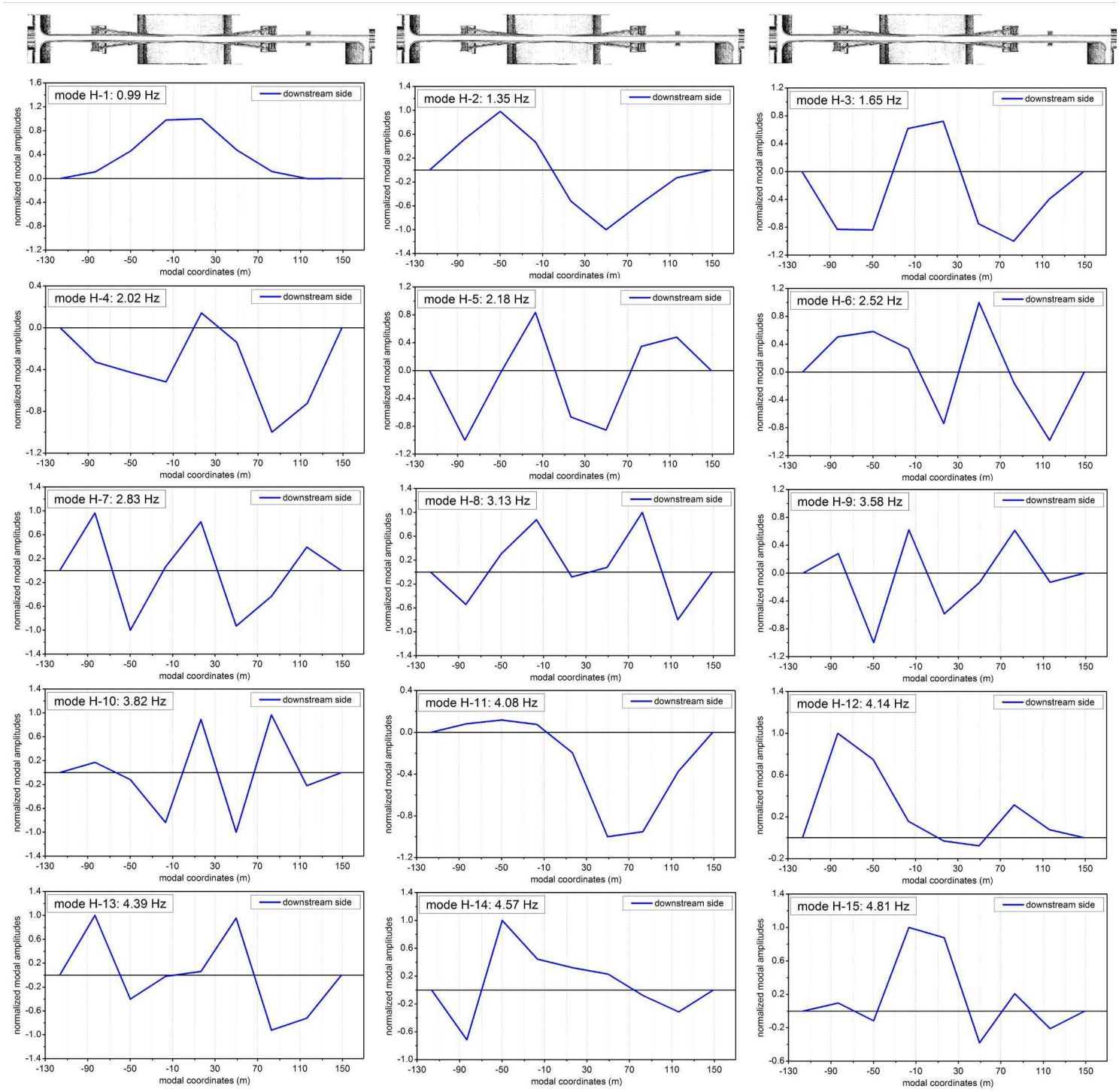
Fig. 11

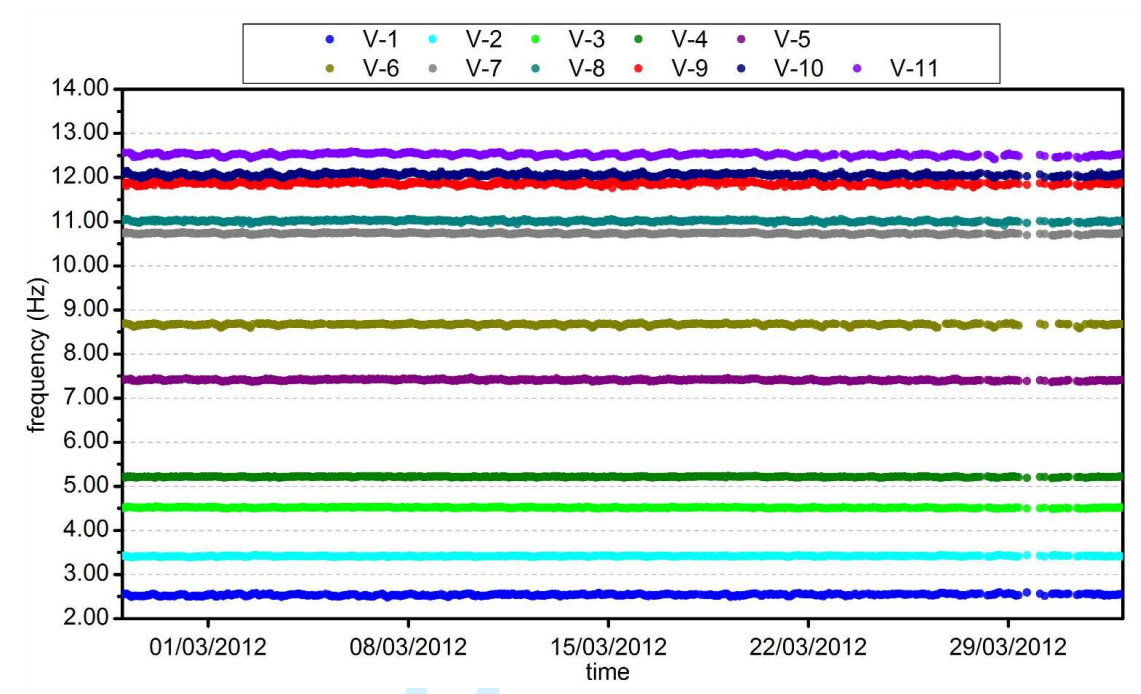


Fig. 12

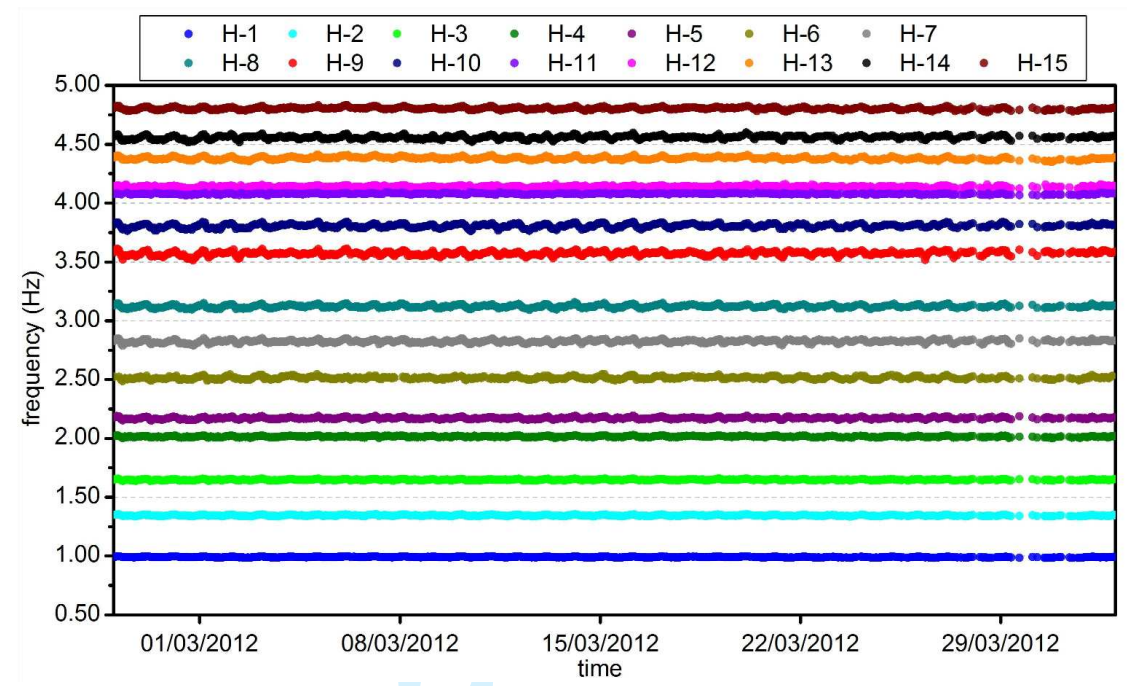

21

22

23

24

25

26

27

28

29

30

31

32

33

34

35

36

37

38

39

40

41

42

43

44

45

46

47

48

49

50

51

52

53

54

55

56

57

58

59 
Fig. 13

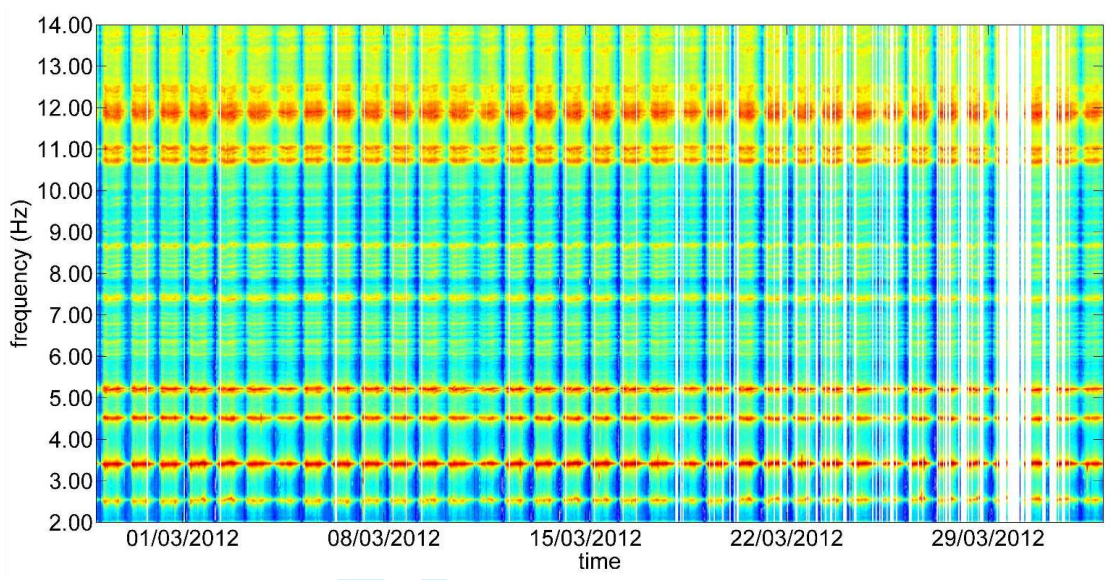


Fig. 14

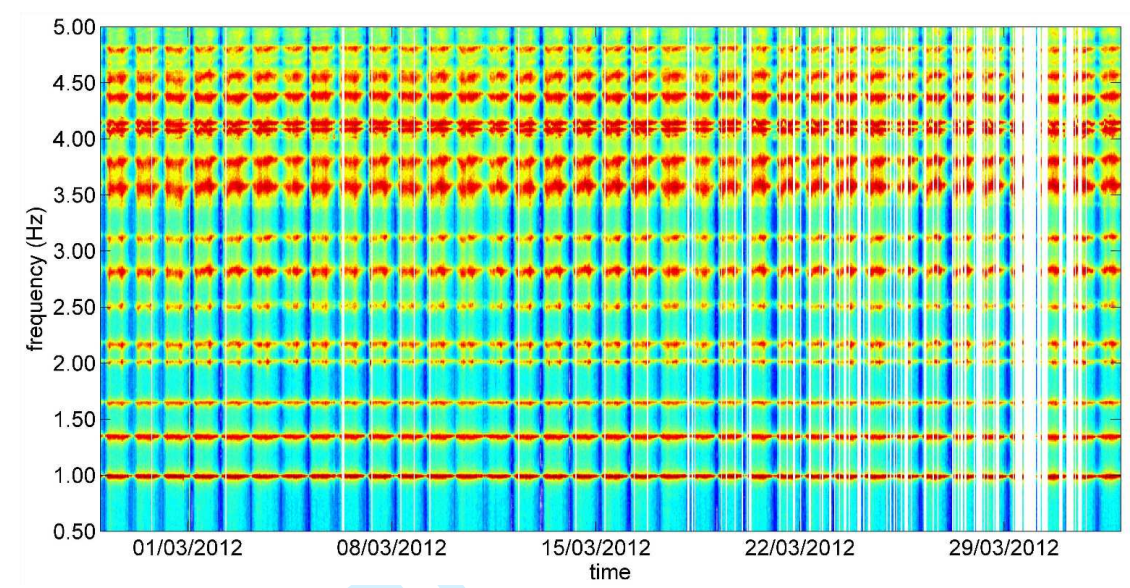


Fig. 15

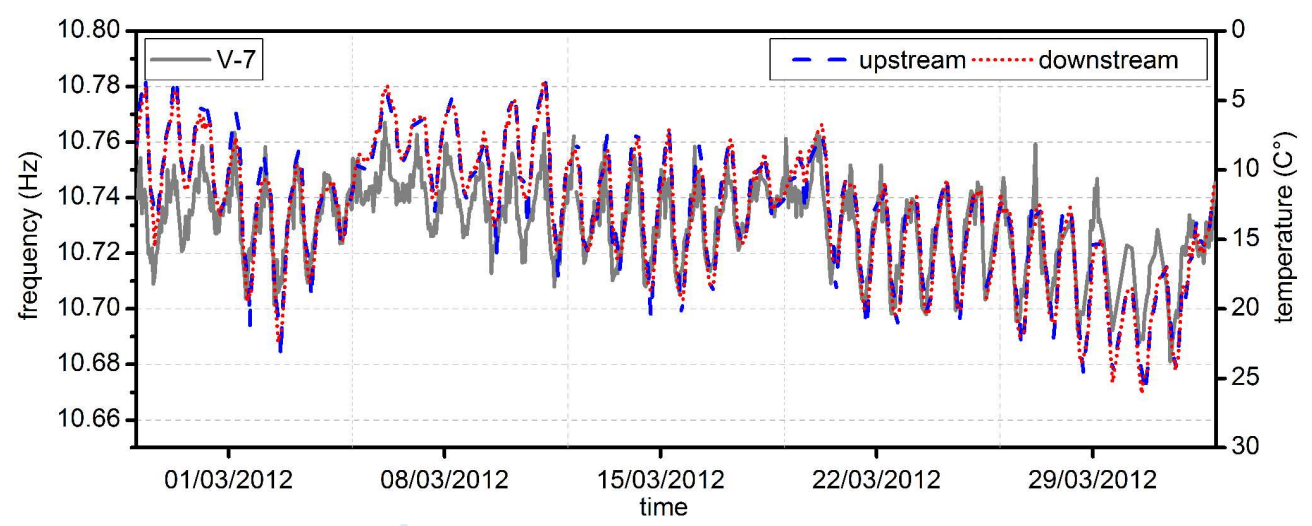

17

18

19

20

21

22

23

24

25

26

27

28

29

30

31

32

33

34

35

36

37

38

39

40

41

42

43

44

45

46

47

48

49

50

51

52

53

54

55

56

57

58

59

60 
Fig. 16

a)

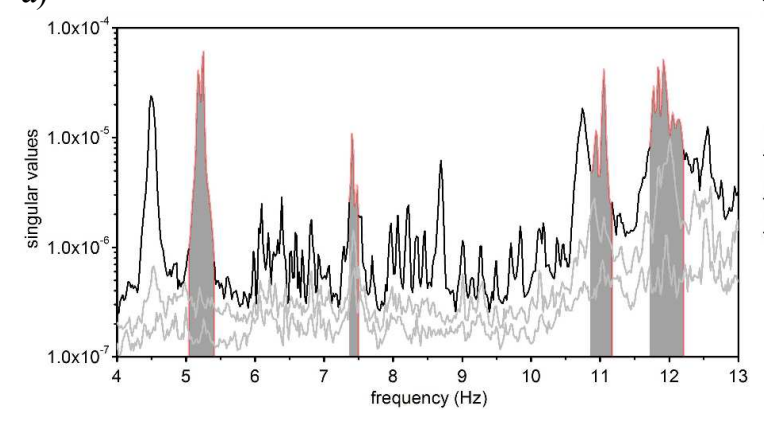

b)

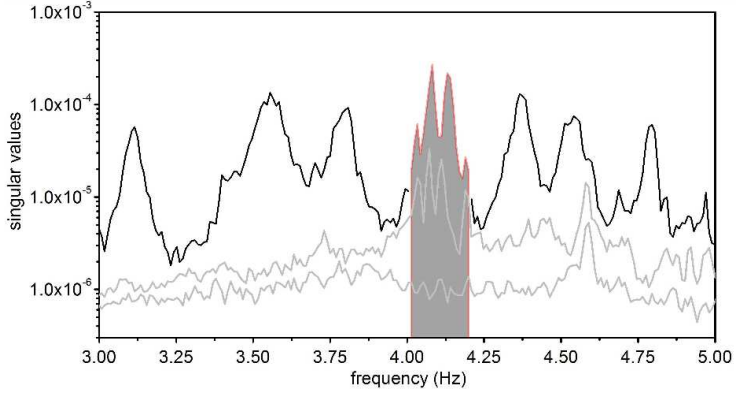

15
16

17

18

19

20

21

22

23

24

25

26

27

28

29

30

31

32

33

34

35

36

37

38

39

40

41

42

43

44

45

46

47

48

49

50

51

52

53

54

55

56

57

58

59

60 
Fig. 17

a)

b)

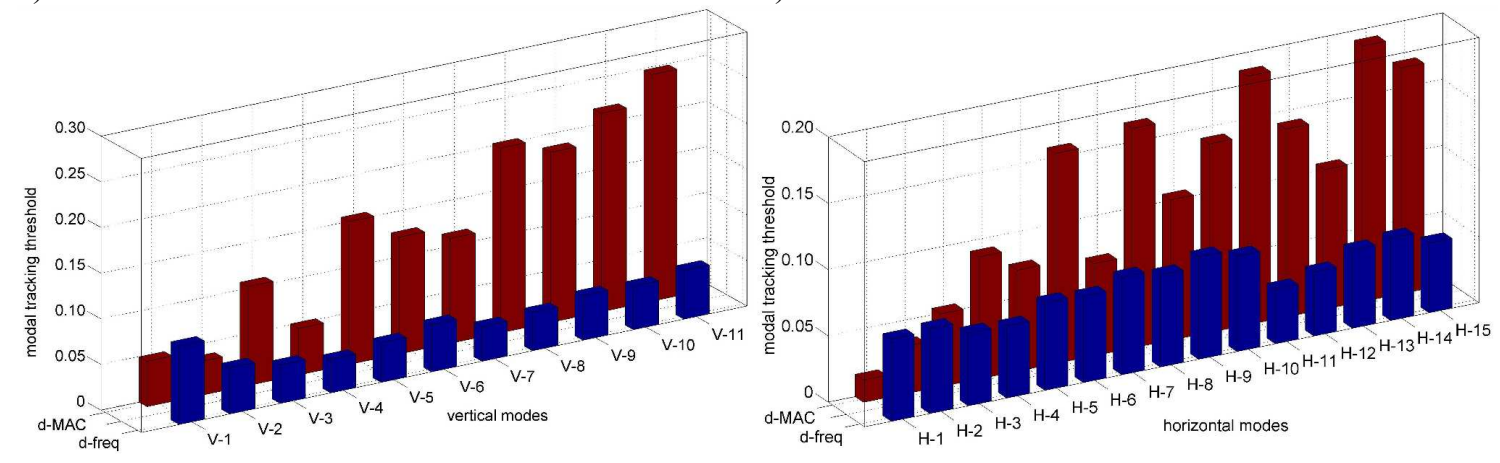



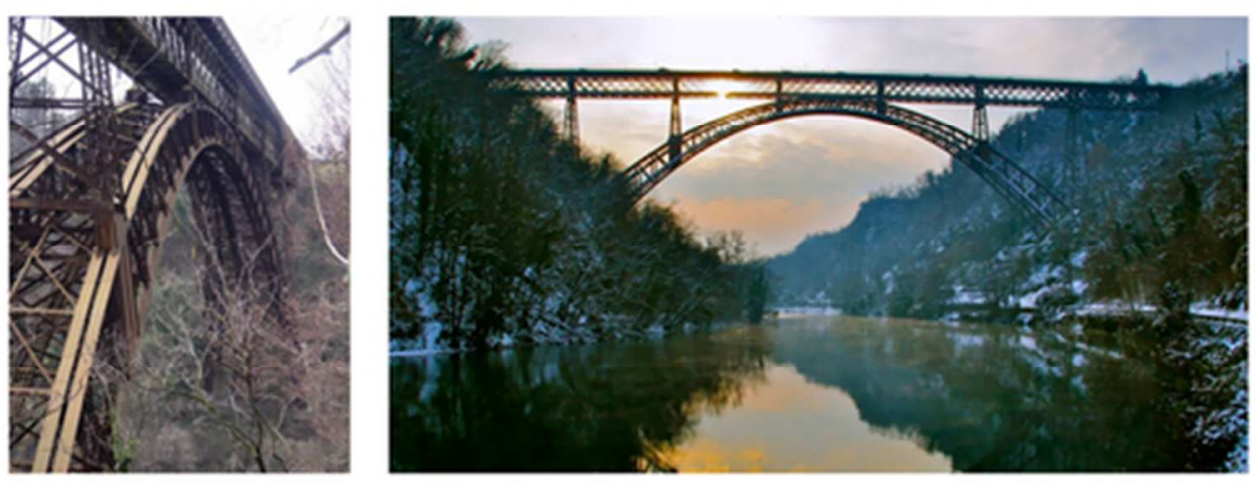

Fig. 1 Views of the San Michele iron arch bridge (1889). $41 \times 15 \mathrm{~mm}(300 \times 300 \mathrm{DPI})$ 

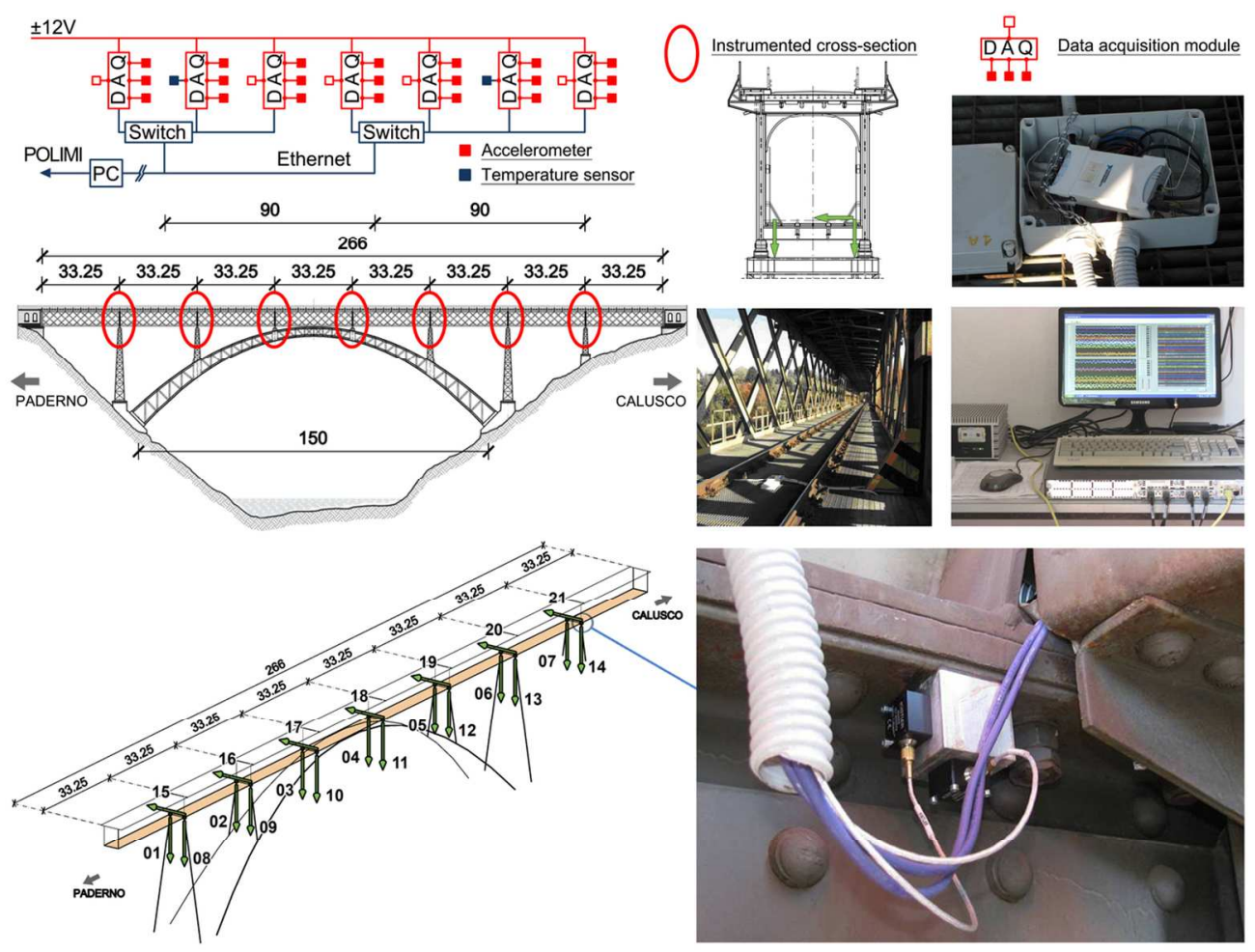

Fig. 2 Layout of the monitoring system. $122 \times 93 \mathrm{~mm}(300 \times 300$ DPI $)$ 


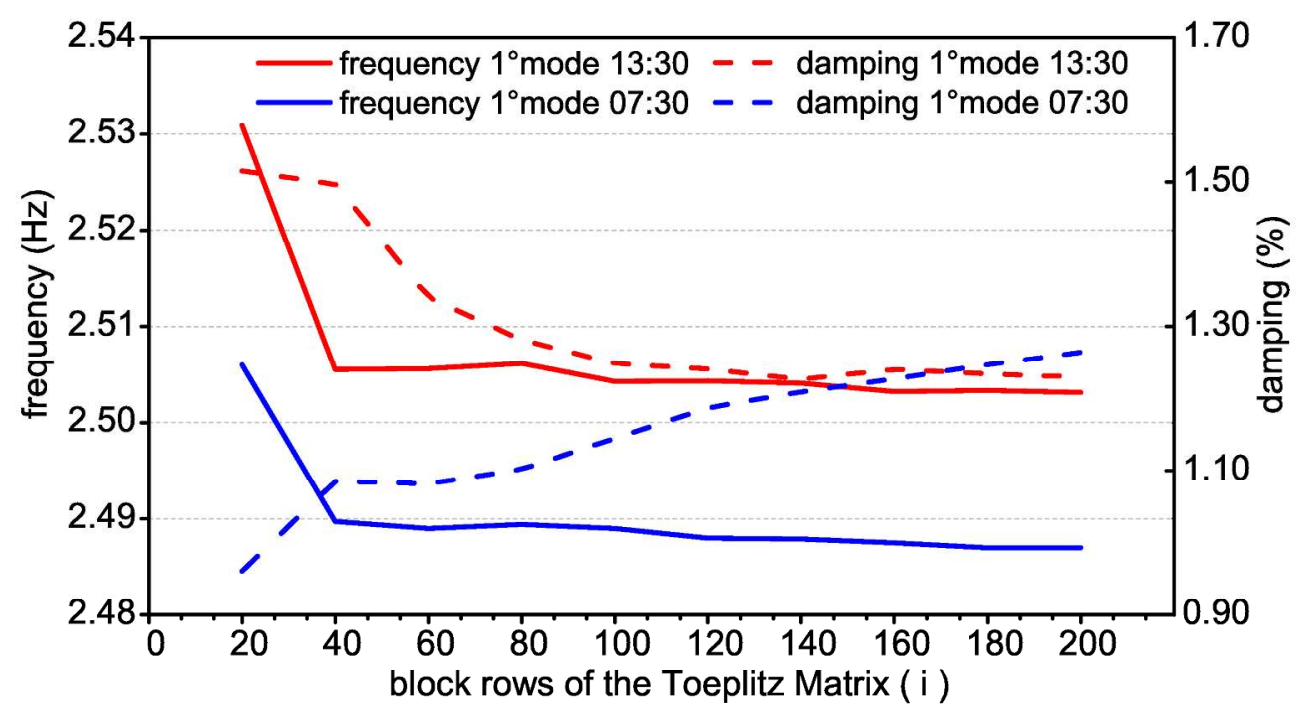

Fig. 3 Effect of the time-lag $i$ on the modal properties of the first vertical mode $\mathrm{V}-1$ (data sets 27/02/2012, $07: 30$ and $13: 30)$. 
a)

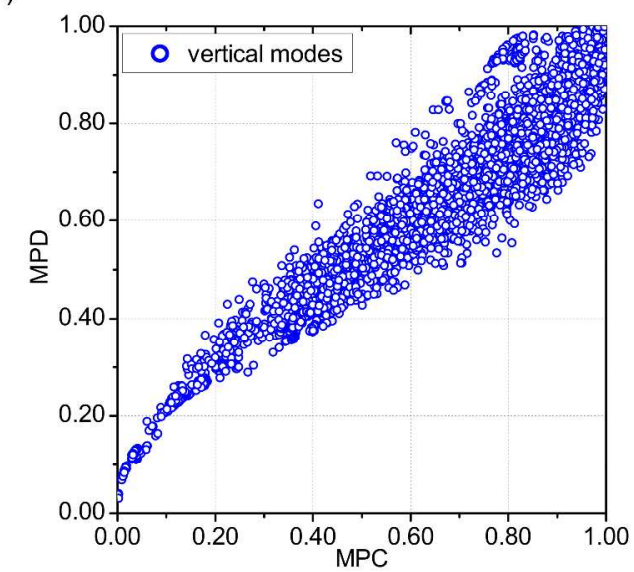

b)

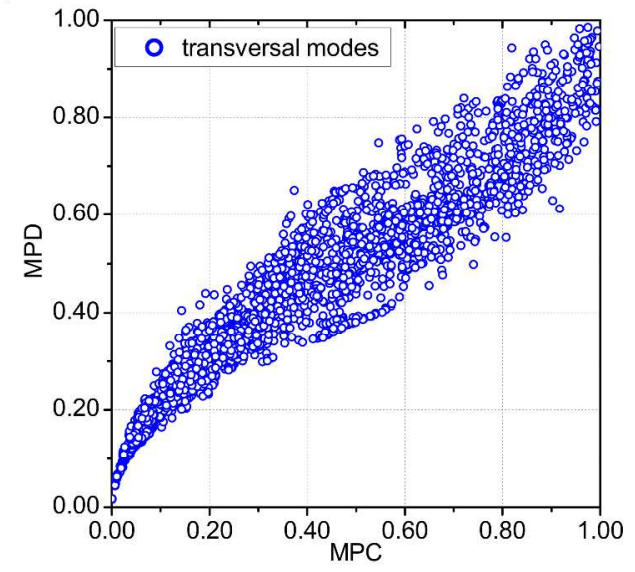

Fig. 4 Consistency check between the MPC and MPD index before clustering: a) vertical modes (27/02/2012, $01: 30)$; b) transversal vibration $(27 / 02 / 2012,00: 30)$. 

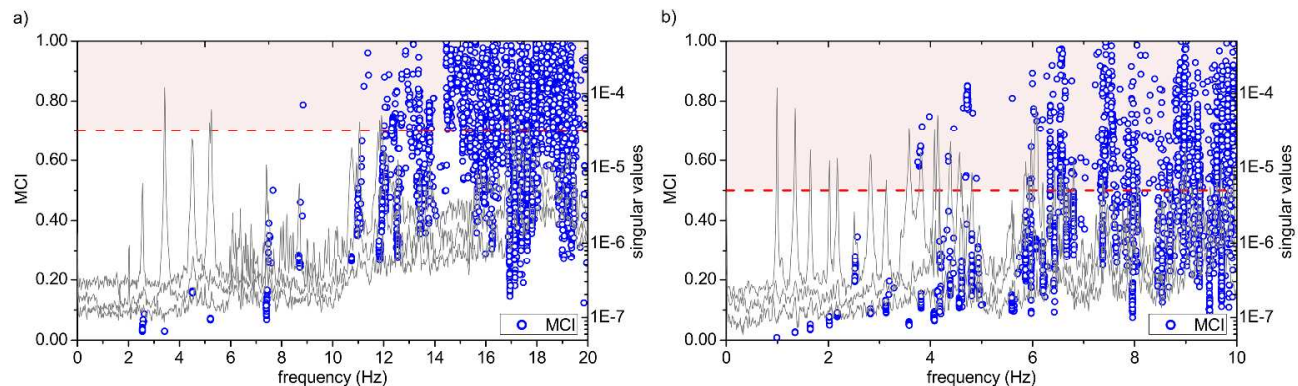

Fig. 5 Plot of natural frequencies of the identifies poles versus the MCI before clustering: a) vertical vibration modes $(27 / 02 / 2012,01: 30)$; b) transversal vibration $(27 / 02 / 2012,00: 30)$. 


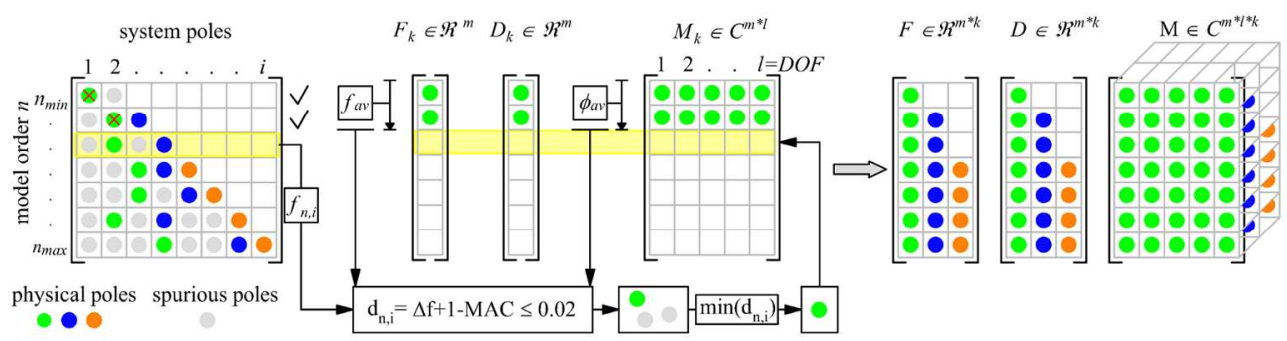

Fig. 6 Sketch of the adopted clustering procedure. $160 \times 42 \mathrm{~mm}(300 \times 300 \mathrm{DPI})$ 

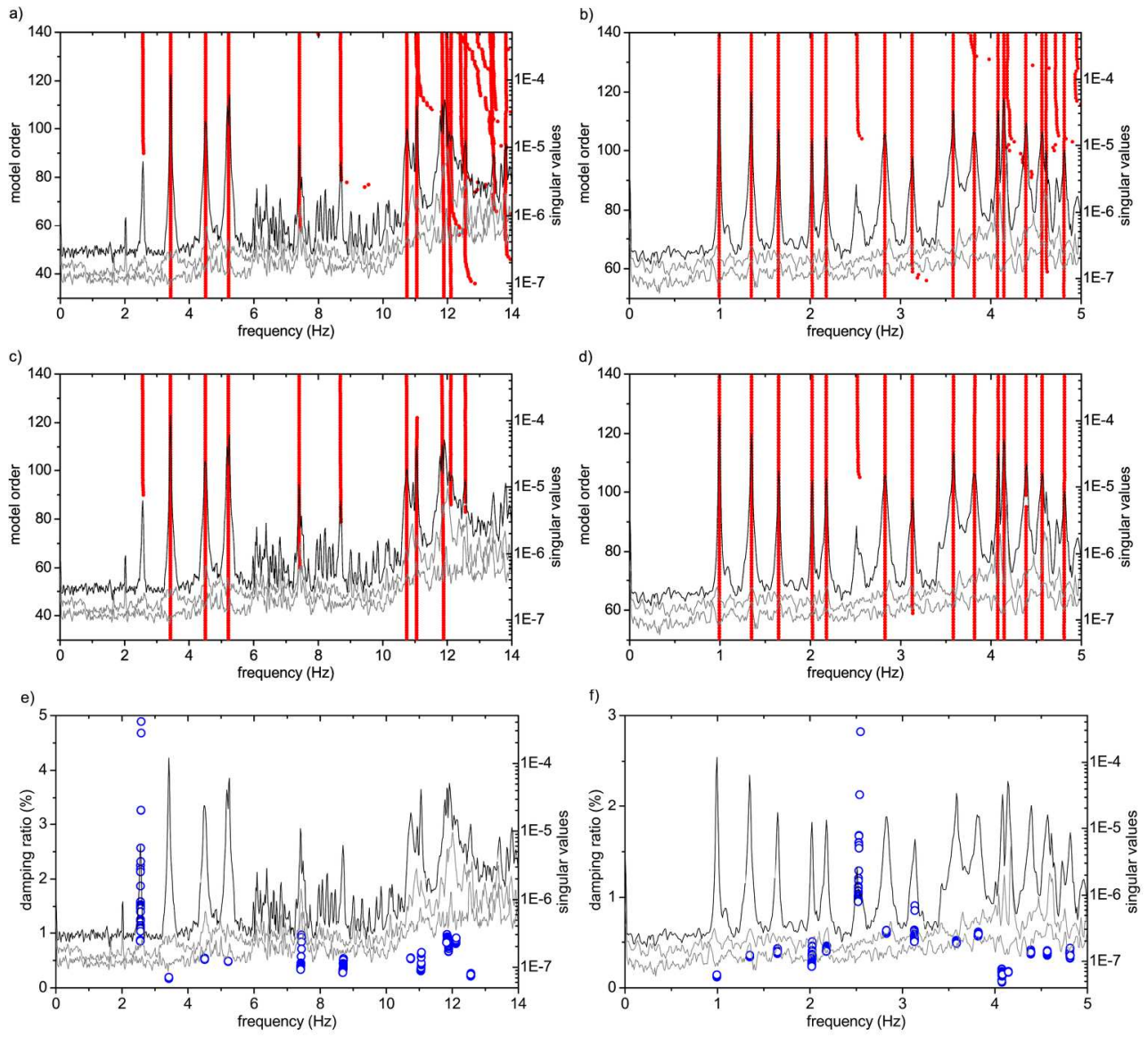

Fig. 7 Vertical modes data set 27/02/201, 01:30: a) stabilization diagram; c) stabilization diagram after clustering; e) damping ratio vs. frequency. Transversal modes data set 27/02/2012, 00:30: b) stabilization diagram; d) stabilization diagram after clustering; f) damping ratio vs. frequency. $183 \times 165 \mathrm{~mm}(300 \times 300$ DPI $)$ 


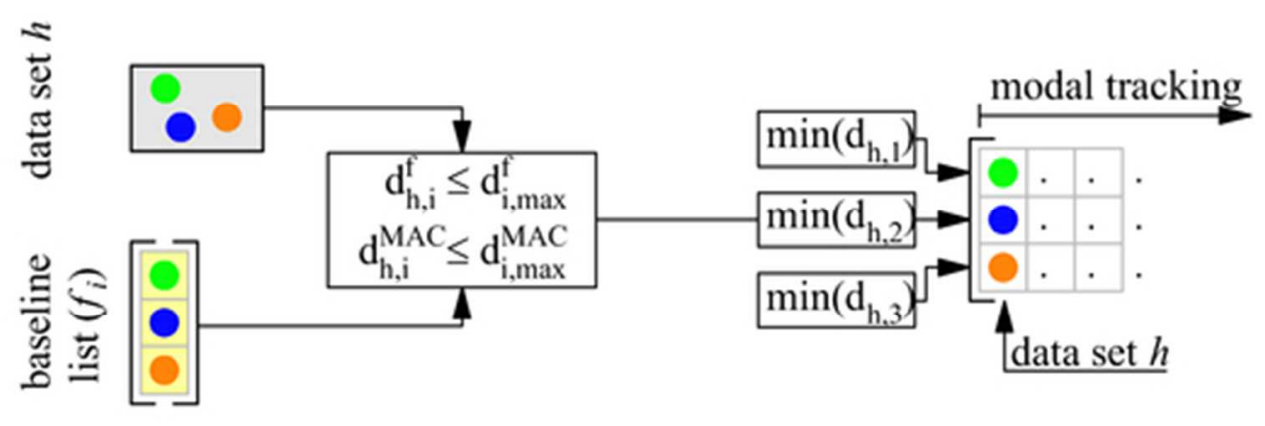

Fig. 8 Sketch of the adopted mode tracking procedure. $45 \times 14 \mathrm{~mm}(300 \times 300$ DPI $)$ 

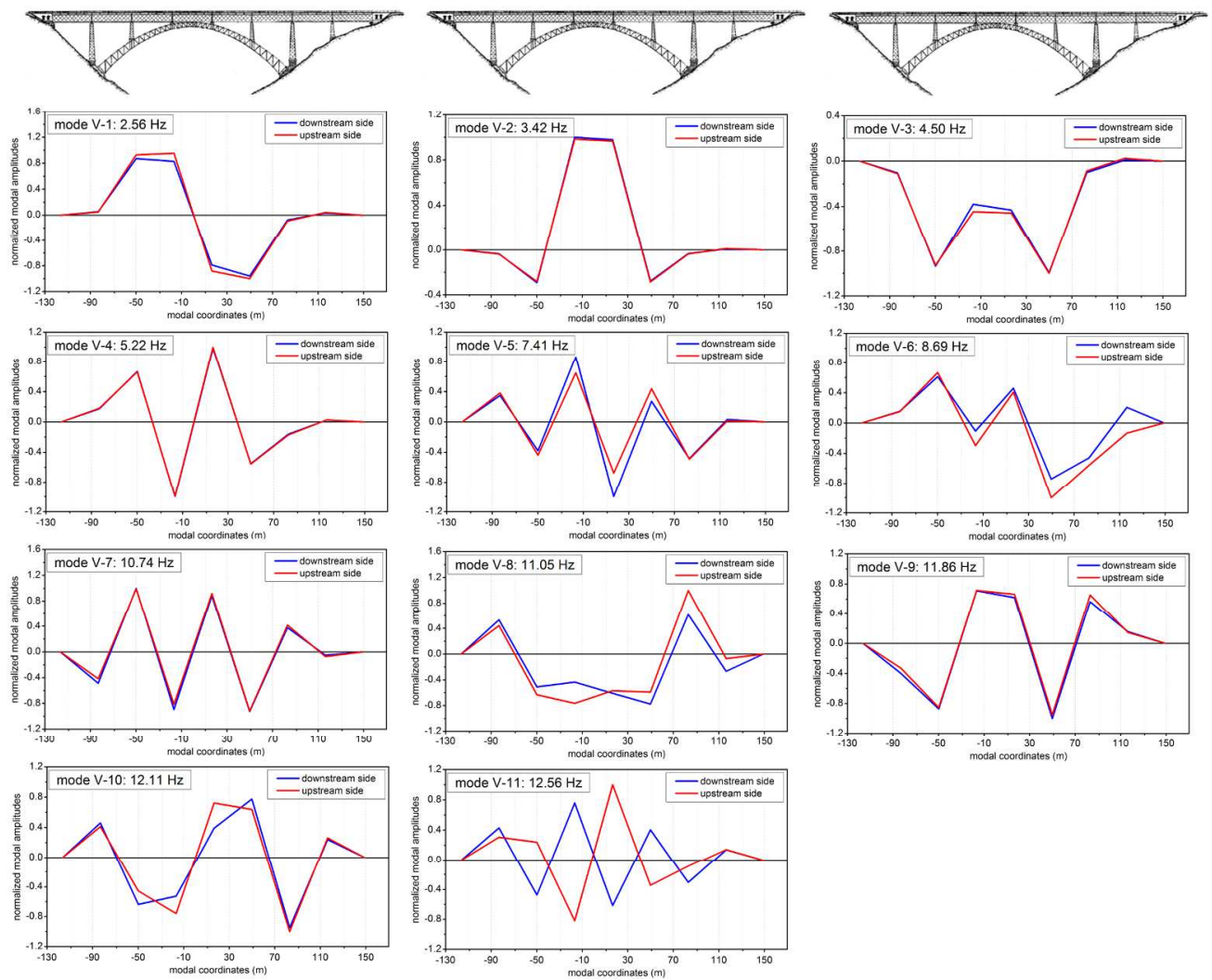

Fig. 9 Baseline vertical modes identified from acceleration measurements with the SSI-Cov procedure $(27 / 02 / 2012,01: 30)$. $187 \times 154 \mathrm{~mm}(300 \times 300 \mathrm{DPI})$ 

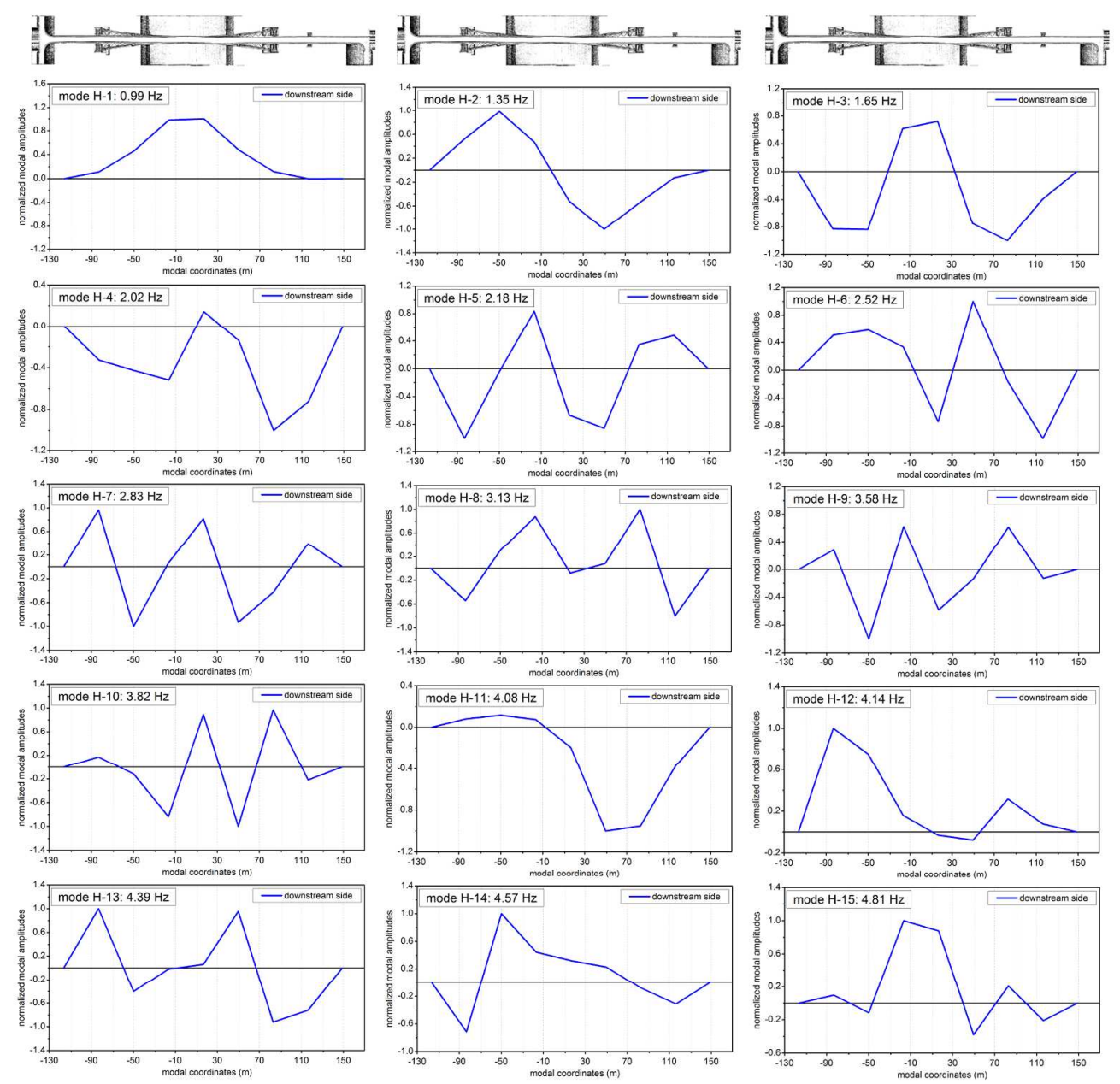

Fig. 10 Baseline transversal modes identified from acceleration measurements with the SSI-Cov procedure $(27 / 02 / 2012,00: 30)$. $188 \times 184 \mathrm{~mm}(300 \times 300 \mathrm{DPI})$ 
Fig. 11 Identified natural frequencies from vertical acceleration measurements using the SSI-Cov application $(27 / 02 / 2012-01 / 04 / 2012)$.

$90 \times 55 \mathrm{~mm}(300 \times 300 \mathrm{DPI})$ 
Fig. 12 Identified natural frequencies from horizontal acceleration measurements using the SSI-Cov application (27/02/2012-01/04/2012). $91 \times 56 \mathrm{~mm}(300 \times 300 \mathrm{DPI})$ 
Fig. 13 First singular value plot of the vertical signals frequency content over time (27/02/201201/04/2012).

$77 \times 39 \mathrm{~mm}(300 \times 300$ DPI $)$ 


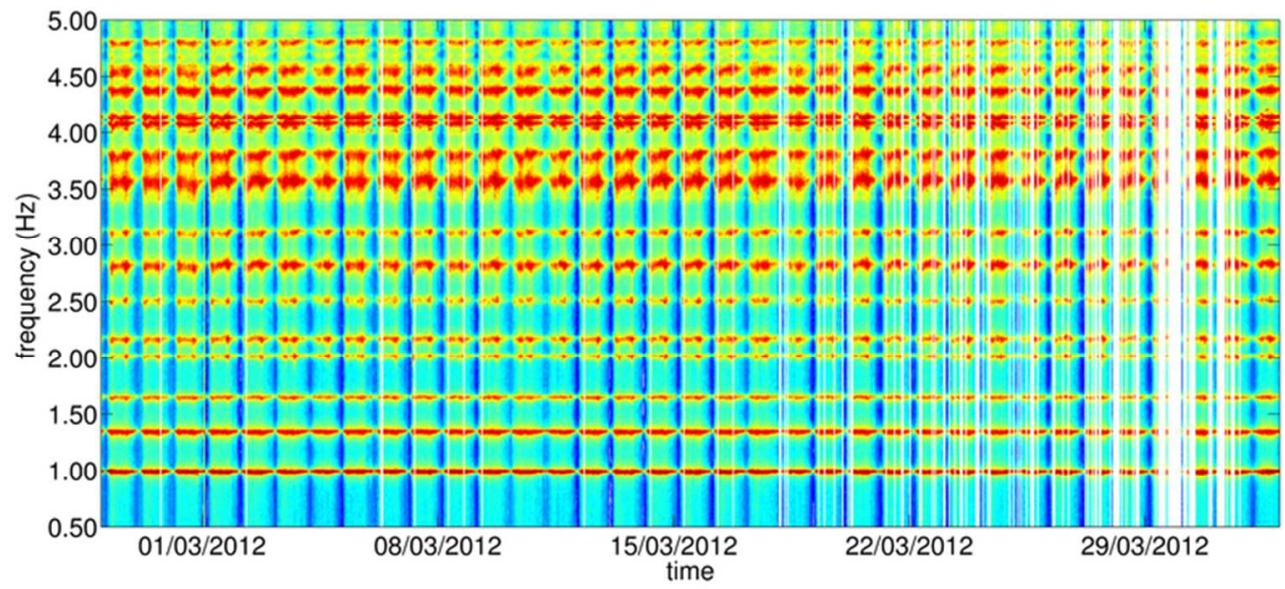

Fig. 14 First singular value plot of the horizontal signals frequency content over time $(27 / 02 / 2012-$ 01/04/2012).

$68 \times 31 \mathrm{~mm}(300 \times 300 \mathrm{DPI})$ 
Fig. 15 Cyclic behaviour of the identified vertical mode V-7 and temperature variation (upstream and downstream temperature sensors). $60 \times 24 \mathrm{~mm}(300 \times 300 \mathrm{DPI})$ 

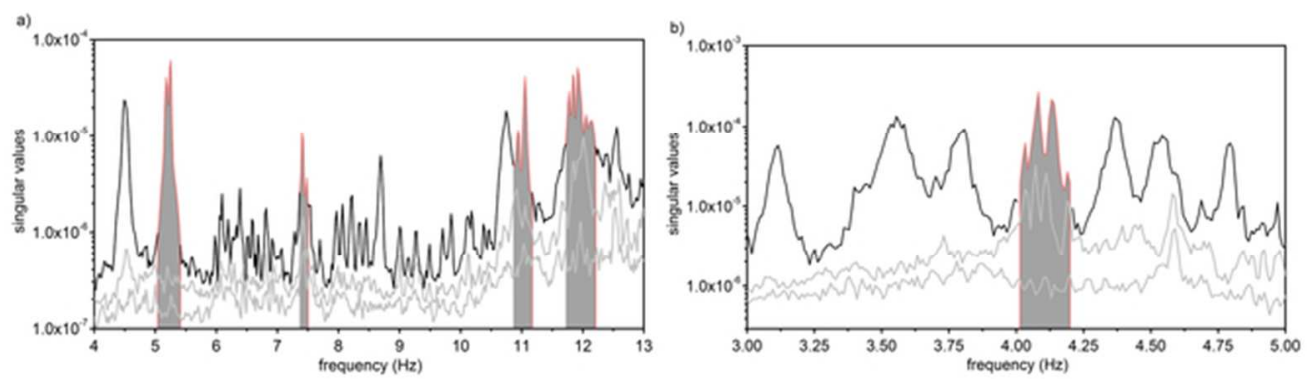

Fig. 16 Zoom of frequency splitting: a) vertical modes (data set 27/02/2012, 01:30); b) horizontal modes (data set 27/02/2012, 17:30).

$56 \times 15 \mathrm{~mm}(300 \times 300 \mathrm{DPI})$

20

21

22

23

24

25

26

27

28

29

30

31

32

33

34

35

36

37

38

39

40

41

42

43

44

45

46

47

48

49

50

51

52

53

54

55

56

57

58

59

60 

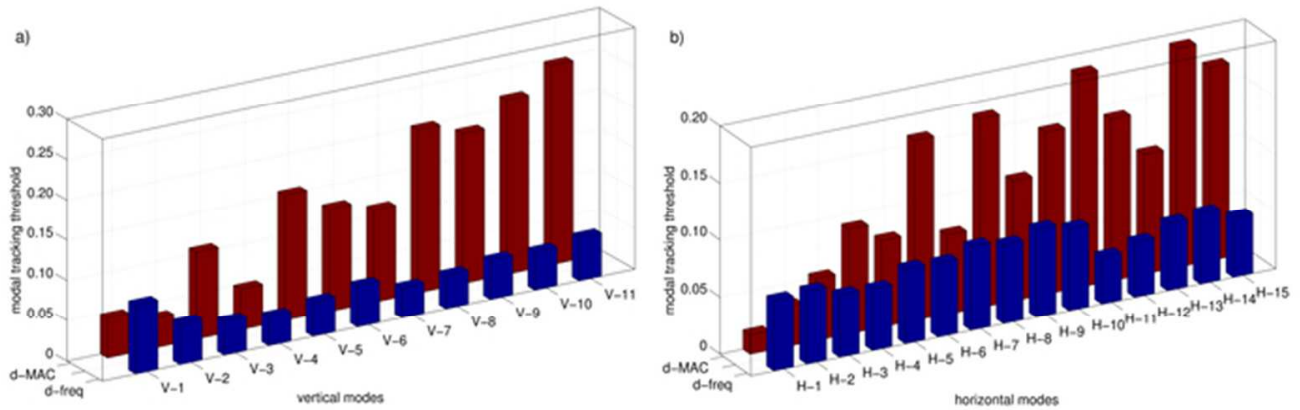

Fig. 17 Adaptive modal tracking threshold for each mode obtained after the final set of data: a) vertical modes; b) horizontal modes. $58 \times 19 \mathrm{~mm}(300 \times 300 \mathrm{DPI})$ 